This document is the Accepted Manuscript version of a Published Work that appeared in final form in The Journal of Physical Chemistry C, copyright $@$ A American Chemical Society after peer review and technical editing by the publisher. To access the final edited and published work see http://pubs.acs.org/articlesonrequest/AOR-Y4Zg7X8QEHynqqCaErXD .

\title{
Effects of Embedded Dipole Layers on Electrostatic Properties of Alkanethiolate Self-Assembled Monolayers
}

Orlando M. Cabarcos, ${ }^{1, a, \#}$ Swen Schuster, ${ }^{2, b, \#}$ Iris Hehn, ${ }^{3, \#}$ Peng Peng Zhang, ${ }^{1, c}$ Masato M. Maitani, ${ }^{1, \mathrm{~d}}$ Nichole Sullivan, ${ }^{1, \mathrm{e}}$ Jean-Benoit Giguère, ${ }^{4}$ Jean-François Morin, ${ }^{4}$ Paul S. Weiss,${ }^{5}$ Egbert Zojer, ${ }^{3,}{ }^{*}$ Michael Zharnikov, ${ }^{2, *}$ and David L. Allara ${ }^{1, *}$

${ }^{1}$ Departments of Chemistry and Materials Science \& Engineering, The Pennsylvania State University, 104 Chemistry Building, University Park, PA 16801-6300, United States

${ }^{2}$ Applied Physical Chemistry, Heidelberg University, Im Neuenheimer Feld 253, D-69120 Heildelberg, Germany

${ }^{3}$ Institute of Solid State Physics, NAWI Graz, Graz University of Technology, Petersgasse 16, 8010 Graz, Austria

${ }^{4}$ Département de Chimie and Centre de Recherche sur les Matériaux Avancés (CERMA), Université Laval, 1045 av. de la Médecine, Québec QC Canada G1V 0A6

${ }^{5}$ California NanoSystems Institute, Department of Chemistry \& Biochemistry, Department of Materials Science \& Engineering, University of California, Los Angeles, CA 90095, United States 


\begin{abstract}
Alkanethiolates (ATs) forming self-assembled monolayers (SAMs) on coinage metal and semiconductor substrates have been used successfully for decades for tailoring the properties of these surfaces. Here, we provide a detailed analysis of a highly promising class of AT-based systems, which are modified by one or more dipolar carboxylic acid ester groups embedded into the alkyl backbone. To obtain comprehensive insight, we study nine different embedded-dipole monolayers and five reference non-substituted SAMs. We systematically varied chain lengths, ester group orientations, and number of ester groups contained in the chain. To understand the structural and electronic properties of the SAMs, we employ a variety of complementary experimental techniques, namely infrared reflection absorption spectroscopy (IRS), highresolution X-ray photoelectron spectroscopy (XPS), ultraviolet photoelectron spectroscopy (UPS), atomic force microscopy (AFM), and Kelvin probe (KP) AFM. These experiments are complemented with state-of-the-art electronic band-structure calculations. We find intriguing electronic properties like large and variable SAM-induced work function modifications and dipole induced shifts of the electrostatic potential within the layers. These observations are analyzed in detail by joining the results of the different experimental techniques with the atomistic insight provided by the quantum-mechanical simulations.
\end{abstract}




\section{Introduction}

Self-assembled monolayers (SAMs) have become an important part of modern nanotechnology and continue to open opportunities to redefine and to adjust a wide range of physical and chemical properties of solid substrates. ${ }^{1-3}$ In particular, the interfacial electronic properties of metal and semiconductor surfaces can be tailored to significant advantage for device applications such as organic solar cells, light-emitting diodes, and organic thin film transistors. ${ }^{4-10}$ The typically employed strategy involves self-assembly of suitably chosen molecules bearing electric dipolar functional groups on an electrode surface. The resulting monolayer modifies the electrode work function and the alignment between its Fermi energy and the electronic levels of the active organic layer. In this way, significant improvements in charge injection during device operation can be achieved. ${ }^{4,8,9,11,12}$ In addition to work function modifications, in the case of metal electrodes, SAMs on semiconductor surfaces can also modulate electric transport of the sub-surface channel via introduction of a local electric field or new surface states. ${ }^{13-16}$ This phenomenon is now utilized in sensor technology and field-effect transistors. ${ }^{13,17-19}$

When employing SAMs for tuning work functions, a functional group with an electric dipole is usually attached to the molecular backbone as the terminal moiety.,20-25 The surface potential and work function $(\phi)$ shift correlate well with the polarity of this moiety, following simple trends and providing a practical recipe for work function engineering. Additional tuning strategies such as varying the dipole associated with the bonding to the substrate by modifying the docking group or altering the molecular backbone ${ }^{26}$ are less frequently applied.

Adjusting the terminal polar group, however, has essential drawbacks since it not only changes the work function of the SAM-covered substrate, but simultaneously can modify associated chemical and physical properties of the SAM-ambient interface. This effect can, for example, significantly change the growth mode of a subsequently deposited semiconductor film, with significant implications for the performance of the entire device. ${ }^{11}$ In addition, terminal groups of the SAM can be modified by the semiconductor growing on them, especially if these groups are reactive. This can then result in a change of their polarity and, consequently, diminish their positive effect. An alternative strategy to avoid the above drawbacks is the embedding of a polar group into the molecular backbone. ${ }^{9,27-29}$ Of particular importance in the context of the present study is the example of thioaromatic SAMs with an embedded pyrimidine moiety. ${ }^{28,29}$ Depending on the 
orientation of this group in the backbone, the work function of the system can be changed by either +0.57 or $-0.42 \mathrm{eV}$ relative to a reference oligophenylene SAM. This variation is achieved without changing the chemistry for docking to the substrate or the chemical composition of the SAMambient interface. ${ }^{28}$ Tuning the work function is, however, not the only effect of the embedded pyrimidine group in these systems, since it also induces a potential discontinuity inside the monolayer. This effect significantly changes the transport properties of the SAM, shifting the transition voltage and resulting in current rectification. ${ }^{30,31}$ The potential discontinuity also shifts the core-level energies in the regions above and below the embedded dipoles relative to each other. These shifts can be observed directly by X-ray photoelectron spectroscopy (XPS), reflected as different binding energies (BEs) for the photoemission peaks associated with both regions. This observation, along with others, ${ }^{32-35}$ lead us to question the generally accepted chemical shift model that assumes that shifts in the core-level BEs in monomolecular films are solely a consequence of different chemical environments of the respective atoms, ${ }^{36}$ with the energy referenced to the Fermi level of the substrate. In contrast, it suggests that electrostatic shifts not related to the immediate chemical environment of an atom are similarly important. Generally, such electrostatic shifts are superimposed on the chemical ones and can under certain circumstances even play a dominant role. ${ }^{37}$

The respective electrostatic effects in photoemission associated with embedded dipoles have also previously been observed for SAMs of mid-chain ester functionalized alkanethiols (ATs) on $\mathrm{Au}(111)$, and were originally explained within a tentative, phenomenological model combined with density functional theory (DFT) calculations of isolated molecules to determine the direction and magnitude of the dipole of the ester group. ${ }^{38}$ This model was based on structural data obtained by a careful characterization of the SAMs by quantitative infrared reflection absorption spectroscopy (IRS), near-edge X-ray absorption fine structure (NEXAFS) spectroscopy, and ancillary ellipsometry. These measurements allowed the establishment of the structure of the molecular chain and the ester group orientations, and yielded information on the film thickness. ${ }^{38}$ Given the success of the above study and considering the recent progress in theory regarding the understanding of electrostatic properties of SAMs, ${ }^{28,29,37,39,40}$ we have decided to revisit mid-chain ester functionalized ATs on $\mathrm{Au}(111)$. The first step along these lines was made by us recently, viz. a detailed theoretical analysis of the C 1s XPS spectra of a representative mid-chain ester 
functionalized monolayer ${ }^{37}$ As a second step, in the present study, we have further expanded both the set of dipole-containing SAM precursors and the types of experiments used to characterize the electrostatic behavior of the SAMs. Most importantly, we performed work function measurements in addition to studying the core-level excitations. The former were done employing two different techniques, namely by determining the secondary electron cutoff in ultraviolet photoelectron spectroscopy (UPS) experiments and by Kelvin probe measurements using non-contact atomic force microscopy (KP-AFM). To provide further insight, the experimental studies were combined with DFT-based simulations of extended metal-SAM interfaces. These simulations go far beyond a simple phenomenological model developed by us in ref 38 .

\section{Experimental methods}

2.1. Studied systems. The studied systems are mid-chain ester functionalized ATs and nonsubstituted ATs serving as references. The non-substituted ATs will be designated as Cn, where $\mathrm{n}$ denotes the number of $\mathrm{CH}_{\mathrm{x}}$ moieties in the alkyl chain. To designate the mid-chain ester functionalized ATs, we use the nomenclature $\mathrm{CmECk}$, where $\mathrm{m}$ and $\mathrm{k}$ denote the numbers of $\mathrm{CH}_{\mathrm{x}}$ moieties below and above the ester group. The $-\left(\mathrm{CH}_{2}\right)_{\mathrm{m}}-$ segment closest to the substrate will be referred to as the bottom segment while the one at the ambient side is considered the top segment. One system containing two ester groups is designated C10EC4EC5 and contains 21 carbon atoms in total with both ester groups aligned in the same direction.

The orientation of the embedded ester group in each system studied has the ester carbonyl group closest to the substrate and the ether oxygen directly above it, with the sole exception of the $\mathrm{C} 10 \mathrm{E}^{*} \mathrm{C} 10$ compound in which the orientation of the ester group has been reversed. As prototypical examples, the structures of $\mathrm{C} 10 \mathrm{EC} 10$ and $\mathrm{C} 10 \mathrm{E}^{*} \mathrm{C} 10$ are shown in Figure 1a, where, respectively, they are characterized by a downward (-z) and upward $(+\mathrm{z})$ tilting of the $\mathrm{C}=\mathrm{O}$ part of the - $\mathrm{COO}-$ unit, which in turn imparts a corresponding downward and upward tilt to the negatively charged end of the dipole. The compounds studied and the abbreviations for their SAMs are listed in Table 1. Note that certain properties of the monolayers of C5EC10, C10EC5, C10EC10, and C15EC5 have already been described in our previous publication ${ }^{38}$ and the SAM of C10EC5 served as a model system to describe the photoemission from the mid-chain functionalized ATs, ${ }^{37}$ 
whereas SAMs of C10EC15, C15EC10, C20EC5, C10E*C10, and C10EC4EC5 are now discussed for the first time.
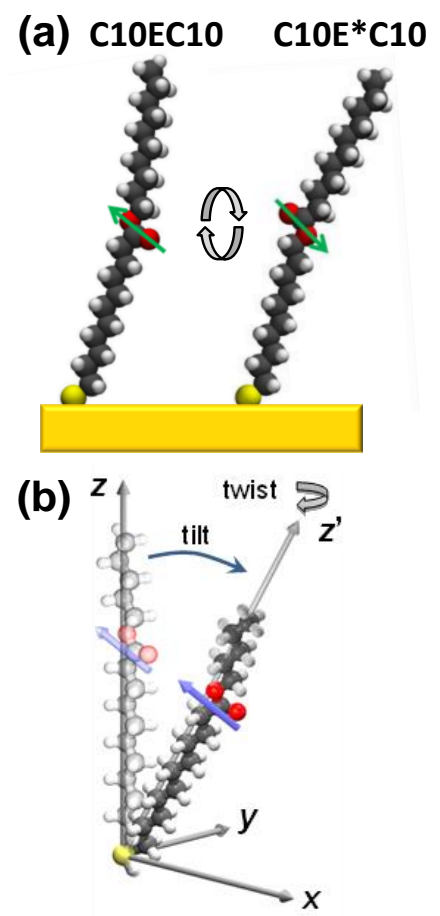

Figure 1. a) Schematic structures of the embedded ester ATs, C10EC10 and C10E*C10 attached to a gold surface in all-trans configuration (yellow: sulfur, gray: carbon, red: oxygen, white: hydrogen). The orientations of the dipole moments are also indicated. b) Definition of the chain tilt and twist angles (note that the carbon backbones and ester groups are assumed to be initially aligned in the $x z$ plane).

2.2. Sample Preparations. Non-substituted ATs were purchased from Sigma-Aldrich and used as received. General details for the synthesis of the mid-chain ester molecules have been described previously. ${ }^{38}$ In brief, the procedures involved a simple esterification of the starting $\omega$ mercaptocarboxylic acid $\left(\mathrm{HSCm}-\mathrm{CO}_{2} \mathrm{H}\right)$ with the selected alkanol $(\mathrm{Ck}-\mathrm{OH})$. In the case of the inverse ester configuration, the $\omega$-mercaptoalkanol $(\mathrm{HSC} 10-\mathrm{OH})$ was used to esterify the alkanoic acid $\left(\mathrm{C} 10 \mathrm{CO}_{2} \mathrm{H}\right)$ and, in the case of the diester, the starting $\omega$-mercaptocarboxylic acid was first esterified with the selected $\omega$-hydroxy acid and then the hydroxyl group of the resulting molecule was esterified with the selected alkanoic acid. Characterization of the molecules was performed using standard methods of infrared spectroscopy and nuclear magnetic resonance. 
For formation of the SAMs, two kinds of substrates were used, viz., vacuum-deposited Au on mica substrates (Agilent/Molecular imaging, Tempe, AZ) and thermally evaporated $\mathrm{Au} / \mathrm{Cr}$ on native oxide covered Si wafers $(200 \mathrm{~nm} / 10 \mathrm{~nm}$ thickness of the metal layers, respectively, $1-1.5 \mathrm{~nm} \mathrm{rms}$ surface roughness of the final $\mathrm{Au}$ film). The former substrates were hydrogen flame annealed before use while the latter ones were used directly after removal from the thermal evaporator. We assume that the properties of the SAMs on these two types of substrates were essentially identical, as e.g. evidenced by the similar values of the work function derived from the UPS and KP-AFM experiments performed on the $\mathrm{Au} / \mathrm{Cr} / \mathrm{Si}$ and $\mathrm{Au} /$ mica supported samples, respectively (see below).

The substrates were immersed into a $0.1 \mathrm{mM}$ thiol solution in absolute ethanol (Pharmco) for 24 h. Upon removal from solution, the surface was thoroughly rinsed with dichloromethane, acetone, and ethanol, and then dried under a nitrogen flow.

2.3. Sample Characterization: General Comments. The mid-chain functionalized and reference AT SAMs were characterized using IRS, high-resolution XPS (HRXPS), UPS, AFM, and KPAFM. All experiments were performed at room temperature. The HRXPS, UPS, and NC-AFM measurements were conducted under ultra-high vacuum (UHV) conditions (base pressure $<7 \times$ $\left.10^{-8} \mathrm{~Pa}\right)$. The spectroscopic and microscopic experiments were performed on the $\mathrm{Au} / \mathrm{Cr} / \mathrm{Si}$ and $\mathrm{Au} /$ mica supported samples, respectively. The IRS, AFM, and KP-AFM experiments were performed on freshly prepared samples. For transport to the synchrotron for the HRXPS and UPS data collection, the freshly made samples were immediately put into clean fluoroware wafer containers and packed in plastic bags sealed under an argon purge for transport. This procedure has proved very effective for maintaining sample integrity. The experiments were complemented by theoretical simulations.

2.4. Infrared Reflection Spectroscopy and Associated Spectral Simulations. Details of the IR instrumentation and spectroscopy procedures have been described earlier. ${ }^{38}$ In brief, reflection spectra were obtained using a customized Fourier transform infrared spectrometer (BioRad FTS7000, Digilab, Randolph, MA) modified to accommodate the sample and detector optics housed in an external $\mathrm{N}_{2}$ purge box and a custom made optical train to produce a $\pm 2^{\circ}$ divergent beam at the sample. The signal was collected by a liquid $\mathrm{N}_{2}$ cooled MCT detector. Spectra were obtained using $p$-polarized light at an $86^{\circ}$ incidence angle with $2 \mathrm{~cm}^{-1}$ resolution and averaged over 800 scans to improve the signal-to-noise ratio. 
Details of the spectral simulation procedures have been previously presented ${ }^{38}$ and the procedures in the present case follow the previous work in exact detail. The simulations utilize a combination of experimental and theoretical data. In brief, isotropic optical function spectra were obtained from transmission spectra of pressed $\mathrm{KBr}$ pellets containing precisely known concentrations of pure, polycrystalline thiols. Vibrational assignments and the determination of transition moment directions were based on DFT calculated spectra for isolated molecules fixed in a planar, all-trans conformation. The isotropic optical function spectra were converted to anisotropic tensor spectra by use of the transition moment directions in molecular coordinates and the tensor spectra were then used to simulate spectra of an assembly of fully extended oriented molecules at selected orientations on the Au surface by using previously reported computational methods based on the electromagnetic theory of plane waves interacting with planar surfaces. ${ }^{41}$

2.5. Photoemission Experiments. HRXPS measurements were performed at the bending magnet beamline D1011 of the MAX IV synchrotron radiation facility in Lund, Sweden, using a SCIENTA SES200 electron energy analyzer. The spectra were recorded in the Au 4f, S 2p, C 1s, and $\mathrm{O} 1 \mathrm{~s}$ regions with a special emphasis on the $\mathrm{C} 1 \mathrm{~s}$ range to monitor the electrostatic effects. All spectra were acquired in normal emission geometry at photon energies (PEs) of either 350 or 580 $\mathrm{eV}$. The binding energy (BE) scale of each spectrum was individually calibrated by setting the BE values relative to the $\mathrm{Au} 4 \mathrm{f}_{7 / 2}$ photoemission peak of the underlying $\mathrm{Au}$ substrate at $84.0 \mathrm{eV} .{ }^{42} \mathrm{The}$ energy resolution was 70-100 meV, which is noticeably smaller than the full width at half maximum (fwhm) of the spectral features relevant in this study. The spectra were fitted by symmetric Voigt functions and either Shirley-type or linear backgrounds. For all samples, the same fit parameters were used for identical spectral regions for a given photon energy. The measurements for most samples were repeated several times, with good agreement between the spectra.

2.6. UPS Experiments. Work functions of the samples were determined by measuring the secondary electron cutoff of the UPS spectra following a standard approach. ${ }^{32}$ The experiments were performed at the Max IV facility, using the same beamline and experimental station as in the case of the HRXPS measurements. The photon energy was set to $50 \mathrm{eV}$. The samples were biased by $-25.6 \mathrm{~V}$ relative to ground so that the low energy portion of the spectrum could be observed. The positions of the cutoffs for the samples were referenced to those of C16/Au and freshly 
sputtered gold. The measurements for most samples were repeated several times, with good agreement between the values. In such cases, the average values are reported.

2.7. AFM Measurements: Lateral Force Imaging. Molecular lattice images were taken in a home-built UHV chamber (a base pressure of $10^{-10}$ Torr) outfitted with a RHK 350 scan head and an RHK SPM 100 electronics system (RHK technologies, Troy, MI) by contact mode AFM with lateral force imaging. The imaging was performed with force modulation probes (PPP-FMR, Nanosensors, Switzerland; cantilever force constant $\sim 2.8 \mathrm{~N} / \mathrm{m}$ ). Typical images were obtained at $\sim 5 \mathrm{nN}$ normal force (including the capillary force between the tip and the sample) at scan rates from 50 to $200 \mathrm{~nm} \cdot \mathrm{s}^{-1}$. Due to the stick-slip friction characteristics, lateral force images can display atomic features that are not necessarily shown in the topography.

2.8. Surface Potential (Kelvin Probe) Measurements. This technique provides a powerful, noninvasive way to obtain spatial surface information complementary to UPS work function data. ${ }^{22,43}$ Experiments were conducted in a Jeol JSPM-4500A AFM system equipped with SPM 100 electronics and a PLL Pro universal AFM controller (RHK technologies, Troy, MI). Details of the experimental setup, associated parameters and equations are given in the Supporting Information.

2.9. Electronic Band-Structure Calculations. To gain insight into the electronic structure of the systems of interest, we performed slab type DFT calculations using the VASP ${ }^{44-47}$ code (Vienna Ab Initio Simulation Package, version 5.3.2). To arrive at the minimum energy configuration we employed a pre-optimization step using molecular dynamics (MD) simulations with the LAMMPS $^{48}$ program package. This was done to avoid a bias regarding the specific orientations of the molecules within the unit cell, bearing in mind the considerable number of degrees of freedom owing to the flexibility of the molecular chains. Further details on the MD runs can be found in the Supporting Information. For the DFT calculations, we chose the PBE exchange-correlation functional $^{49}$ including van-der Waals interactions via the PBE+vdW ${ }^{\text {Surf }}$ method $^{50}$ in the implementation of Al-Saidi et al. ${ }^{51}$ The projector-augmented wave (PAW) ${ }^{52,53}$ formalism was used to describe core-valence interactions. A plane wave basis set with a cutoff energy of $400 \mathrm{eV}$ and $6 \times 6 \times 1$ Monkhorst-Pack ${ }^{54}$ k-point grid were employed in all calculations. For geometry optimizations, we combined VASP with the GADGET ${ }^{55}$ tool.

The studied systems were modelled in a $(2 \sqrt{3} \times 3)$ rect surface unit cell containing four molecules consistent with literature reports on alkyl thiolates on gold substrates. ${ }^{56-58}$ We chose this system 
set-up over the also reported $(\sqrt{3} \times \sqrt{3}) \mathrm{R} 30^{\circ}$ surface unit cell containing only one molecule to allow for a possible herringbone arrangement of the molecules (which was, in fact then also obtained in all our geometry optimizations). Structures consistent with the choice of the unit cell have been observed in the AFM experiments (see below). The gold substrate was modeled using five layers, of which the top two were kept mobile during the geometry optimization. To electrostatically and quantum-mechanically decouple periodic replicas of the slab, a self-consistent dipole correction in the $z$-direction was used ${ }^{59}$ combined with a vacuum gap of $25-30 \AA$ between the slabs.

Core-level energies were computed following the initial state approach ${ }^{60}$ which is most appropriate for the present systems, as argued in ref 37. Screening by the metal was accounted for by an image charge model. For weighing the contributions of core-level excitations from different carbon atoms accounting for the finite escape depth of the photoelectrons, an exponential damping of the signal was applied. ${ }^{61}$ Further details on the quantum-mechanical simulations and the approach for modelling the HRXPS experiments are contained in the Supporting Information and in ref 37.

\section{Results}

\subsection{Structure of the mid-ester SAMs}

\subsubsection{Infrared Vibrational Spectroscopy}

The IR spectra of the C5EC10, C10EC5, C10EC10, and C15EC5 SAMs have already been presented and analyzed in detail in ref 38 , along with the spectra of their partly deuterated analogues. In that study the orientations of the alkyl chain segments and the embedded ester moiety were deduced from the best fits of spectral simulations to experimental IRS data as well as from the analysis of NEXAFS spectra, with the isotope substitutions in the IRS case to distinguish between the spectral signatures of the top and bottom segments. ${ }^{38}$ The results of both procedures agreed within experimental error to give an overall average alkyl chain tilt from the surface normal of $31( \pm 4)^{\circ}$ and a chain twist around the long axis of $60( \pm 5)^{\circ}$ (for a definition of those angles see Figure 1b). Note that the above values, which are very similar to those for the Cn SAMs on $\mathrm{Au}(111)$, are more descriptive of the bottom - $\left(\mathrm{CH}_{2}\right)-$ segments which span between the substrate and the ester group. These segments exhibit high conformational ordering, which, within experimental error, is identical to that reported previously for the analogous alkyl chains in $\mathrm{Cn}$ 
SAMs on $\mathrm{Au}(111) \cdot{ }^{38}$ In contrast, the top segments of the mid-ester SAMs are somewhat more conformationally disordered ${ }^{38}$ though they remain generally aligned in an extended, all-trans form with some gauche defects. The alignment of the top segment is also further perturbed by the presence of the ester group itself which can add an additional negative or positive tilt of $\sim 9^{\circ}$ to the top chain depending on the orientation of the ester group (Figure 1) ${ }^{38}$ In addition, the data in ref 38 suggest that the $\mathrm{COO}$ group is roughly coplanar with the $\mathrm{C}-\mathrm{C}-\mathrm{C}$ plane of the bottom and top $-\left(\mathrm{CH}_{2}\right)$ - segments (notwithstanding a certain degree of conformational disorder for the top segment).

In the present study, similar IR data were obtained for the C10EC15, C15EC10, C20EC5,

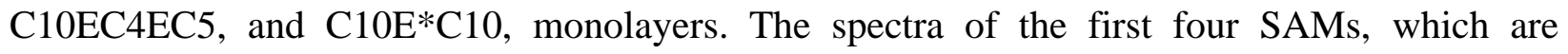
characterized by a downward orientation of the negative end of the embedded dipole (see Figure 1), are presented in Figure 2, along with the spectra of the previously studied monolayers, all of which have similar orientation of the embedded dipole (C5EC10, C10EC5, C10EC10, and $\mathrm{C} 15 \mathrm{EC} 5)^{38}$. The spectral shapes as well as peak intensities and positions for the new and previously studied SAMs closely match each other in both the low and high frequency regions of the spectra. This result indicates that the main structural aspects determined in ref 38 for the orientation and conformational ordering of the previously studied SAMs are also applicable to the new systems. For this reason, detailed spectral simulations were not performed for these monolayers.

In particular, note that in the high frequency $\mathrm{C}-\mathrm{H}$ stretching region (see Figures $2 \mathrm{~b}$ and $2 \mathrm{~d}$ ), which is sensitive to the alkyl chain conformations and tilt angles, the similarity of the general peak envelope, relative intensity patterns, and the peak positions establish the close similarity of the eight SAM structures. As expected, the absolute intensities of the peaks for systems with a larger number of $-\mathrm{CH}_{2}-$ groups are higher. 

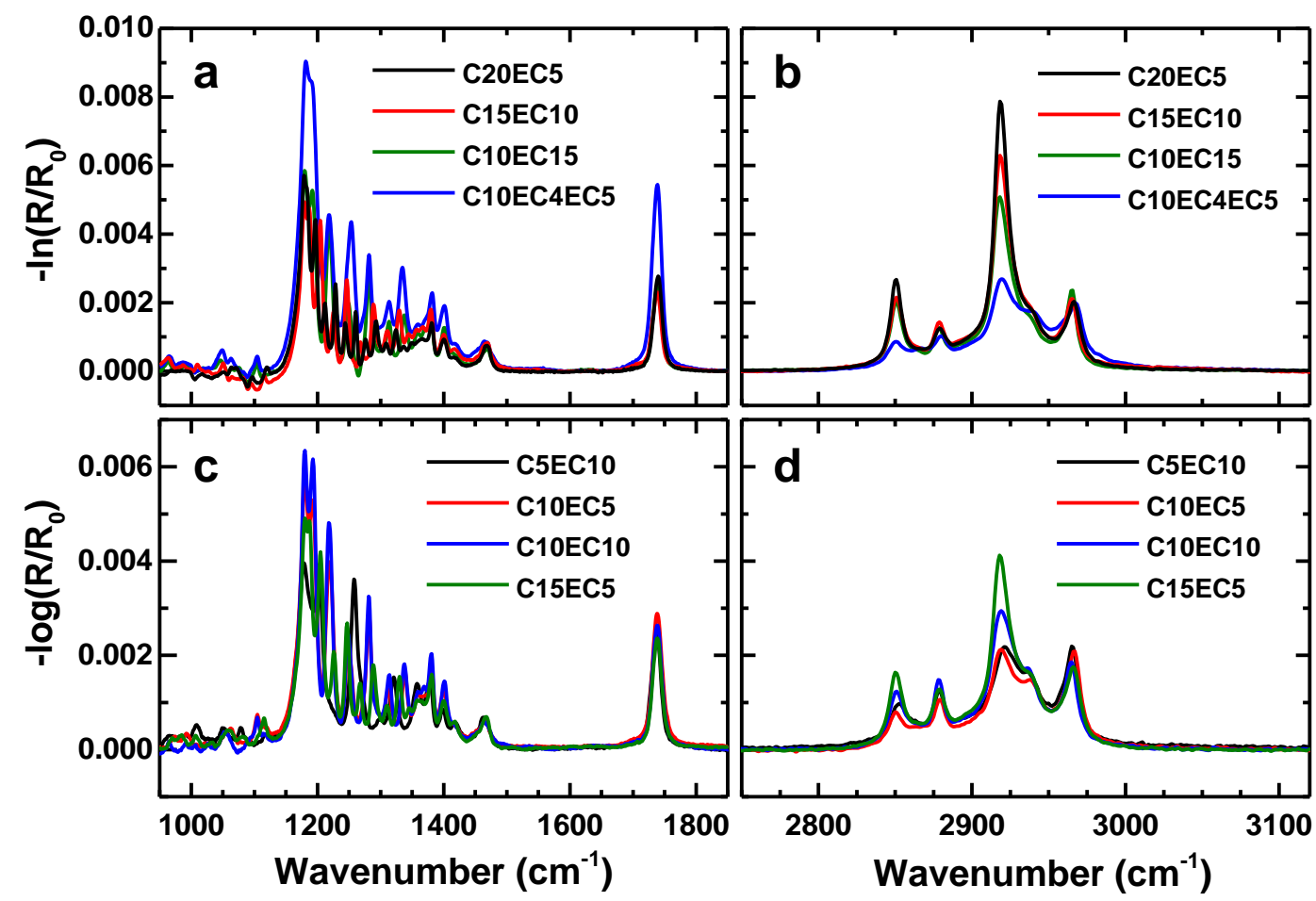

Figure 2. Overlaid IR spectra of the ester SAMs with the downward direction of the negative end of the dipole of the embedded ester group in the low ( $\mathbf{a}$ and $\mathbf{c})$ and high (b and $\mathbf{d}$ ) frequency regions, including the data for the new SAMs (a and b) and the monolayers from the previous study (c and d).

The low-frequency region (see Figures 2a and 2c) is more complicated due to the appearance of regularly spaced sharp peaks between $\sim 1200$ and $1400 \mathrm{~cm}^{-1}$, which are assigned to the $\mathrm{CH}_{2}$ twisting and wagging mode progression bands. These peaks are generally strongest for all-trans conformation segments when these segments are electronically coupled to a polar functional group, such as an ester. Consequently, in the given case, these progression bands dominantly arise from the bottom segments, which are more ordered than the top ones and have a better electronic coupling to the ester moiety. ${ }^{38}$ The number of peaks in a progression is a direct function of the number of connected - $\left(\mathrm{CH}_{2}\right)$ - units in a segment so one cannot directly overlay the progression mode peak patterns for ester SAMs with different bottom segment lengths. Nevertheless, the overall spectral envelopes across the wavenumber scale are very similar for both already studied and newly discussed ester SAMs, particularly for the $\mathrm{C}-\mathrm{O}$ and $\mathrm{C}=\mathrm{O}$ stretching mode peaks $(\sim 1175$ and $\sim 1740 \mathrm{~cm}^{-1}$, respectively), which further corroborates that the structures of the present SAMs are essentially equivalent to those in the previous study. In this regard note that the C10EC4EC5 
diester with two ester units has nearly double size intensities for the $\mathrm{C}-\mathrm{O}$ and $\mathrm{C}=\mathrm{O}$ stretching modes, as expected. Further, note that the near superposition of the $\mathrm{C}=\mathrm{O}$ stretching mode peaks for all the single ester SAMs indicates that the $\mathrm{C}=\mathrm{O}$ moieties have very similar orientations relative to the substrate surface.

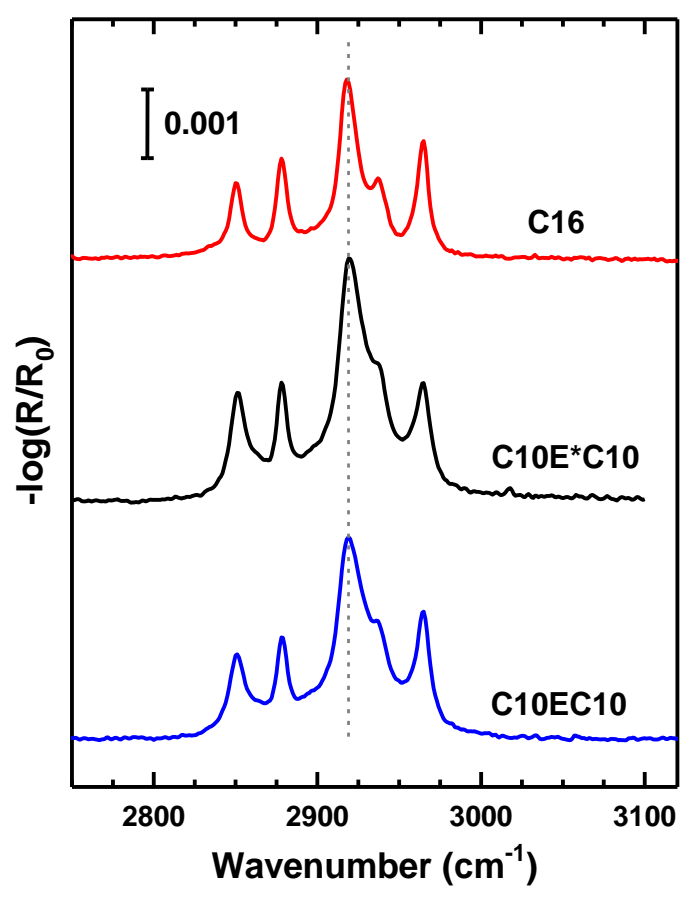

Figure 3. Comparison of the high frequency region IR spectra for the $\mathrm{C} 16, \mathrm{C} 10 \mathrm{E}^{*} \mathrm{C} 10$ and the C10EC10 ester SAMs. A vertical gray dashed line is drawn through the peaks of the antisymmetric $\mathrm{CH}$ stretching mode to illustrate the close positions of the peak frequencies.

In the case of the $\mathrm{C} 10 \mathrm{E}^{*} \mathrm{C} 10$ reverse ester $\mathrm{SAM}$ with the inverted orientation of the embedded ester group (an upward orientation of the negative end of the dipole; see Figure 1), further analysis is required. A direct comparison of the high-frequency $\mathrm{C}-\mathrm{H}$ stretching region spectrum with those of the analogous C10EC10 SAM from our previous study and a standard C16 SAM, are presented in Figure 3. The close correspondence of the peak frequency and intensity patterns establishes that the general conformational ordering and tilt angles of the alkyl chains are quite similar. In particular, note that the peak position of the $\mathrm{CH}$ antisymmetric $\left(\mathrm{d}^{-}\right)$mode at $\sim 2918 \mathrm{~cm}^{-1}$, which is highly sensitive to alkyl chain conformational ordering, is virtually identical for all three SAMs (see dashed line in the figure). From this we conclude that the inverted orientation of the embedded 
ester group does not have any significant effect on the overall alkyl chain orientation and ordering compared to the other SAMs in the present study.

This latter point is established in more detail by a simulation-experiment comparison for the low frequency region spectrum, shown in Figure 4. The observation of a well-defined progression of coupled wag-twist modes in the experimental spectrum of the C10E*C10 SAM shows evidence for a conformationally ordered chain segment. Analysis of this pattern shows that the spacing of the modes is $\Delta v=33.3 \pm 3.3$ which closely corresponds to a $-\left(\mathrm{CH}_{2}\right)_{9}-$ chain which has a predicted spacing of $\Delta v=32.6{ }^{38}$ This allows assignment of the progression modes to the top $-\left(\mathrm{CH}_{2}\right)_{9}-$ segment which was confirmed via DFT calculations of the normal modes of an isolated $\mathrm{C} 10 \mathrm{E}^{*} \mathrm{C} 10$ molecule. Based on this result and the earlier analysis of the CmECk SAMs, it appears that whichever chain segment is directly bonded to the carbonyl carbon of the ester group has the best electronic coupling of the $\mathrm{CH}_{2}$ wag-twist dipole transition moments to the $\mathrm{C}=\mathrm{O}$ stretch mode to yield the observed series of progression bands.

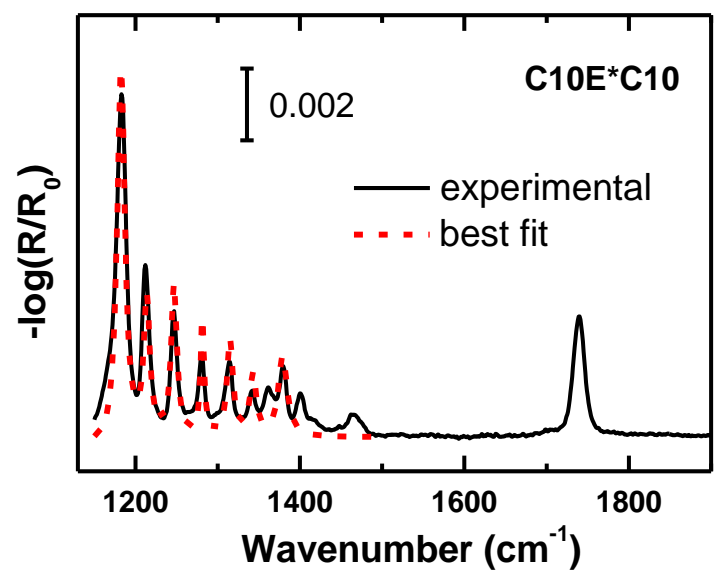

Figure 4. Experimental IR spectrum of the C10E*C10 SAM (black solid line) as well as its bestfit simulation (red dashed line). The sequence of sharp peaks running from just above 1200 to $\sim 1400 \mathrm{~cm}^{-1}$, corresponds to the wag-twist modes. The number of the peaks and their frequency spacings correspond exactly to a conformationally ordered top $-\left(\mathrm{CH}_{2}\right)_{9}-$ segment. The simulation gives a tilt of the top segment of $35^{\circ}\left( \pm 2^{\circ}\right)$, from the surface normal. For details, see text.

A set of simulations was made starting with pure polycrystalline $\mathrm{C} 10 \mathrm{E}^{*} \mathrm{C} 10$ ester to generate isotropic optical functions and the optical tensor spectra were then generated based on the geometry of the fully extended molecule as determined from DFT calculations. The simulations 
were restricted to the progression mode region in order to keep the analysis simple since the geometry of the ester group is more difficult to interpret. In the simulations, the tilt angle of the conformationally ordered $-\left(\mathrm{CH}_{2}\right)_{9}-$ top segment chain was varied. Since the transition dipole moment of the wag-twist modes is aligned parallel to the chain axis, the tilt angle from the surface normal could be determined directly from the intensities. The resultant best-fit corresponds to a tilt angle of $35^{\circ}\left( \pm 2^{\circ}\right)$. The DFT calculations also indicate that the chain axes of the top and bottom alkyl segments of the $\mathrm{C} 10 \mathrm{E}^{*} \mathrm{C} 10$ diverge by $\sim 7^{\circ}$ which translates to a bottom chain tilt of $\sim 28^{\circ}$ $\left( \pm 2^{\circ}\right)$ which is in excellent agreement with the measured tilts of non-substituted AT SAMs and the ester-substituted monolayers described in ref 38. The chain twist angle around the long axis cannot be determined directly from this fit but, based on the similarity of the high frequency region IR spectrum of the $\mathrm{C} 10 \mathrm{E}^{*} \mathrm{C} 10 \mathrm{SAM}$ with those of the C10EC10 monolayers and Cn SAMs on $\mathrm{Au}(111)$ (see Figure 4), the twist angle is estimated to be close to $55-60^{\circ}$.

\subsubsection{AFM Analysis}

The similarity of the basic orientational parameters of the ester containing SAMs and the nonsubstituted Cn systems suggests a uniform translational arrangement in both the standard (CmECk) and reverse ester layers. To verify this hypothesis, lateral force AFM measurements were performed, taking $\mathrm{C} 10 \mathrm{EC} 10$ and $\mathrm{C} 10 \mathrm{E}^{*} \mathrm{C} 10$ monolayers as representative examples. Typical images for these monolayers are shown in Figure 5. Both images confirm an ordered arrangement with the adsorbates in a $(\sqrt{ } 3 \times \sqrt{ } 3) \mathrm{R} 30^{\circ}$ hexagonal lattice with the expected $\sim 0.50 \mathrm{~nm}$ nearest neighbor spacing. This corresponds to a surface density of $\sim 4.60$ molecules $/ \mathrm{nm}^{2}$. Such a lattice is typical of $\mathrm{Cn}$ SAMs on a $\mathrm{Au}(111)$ surface, where a $\mathrm{c}(4 \times 2)$ modulation of the $(\sqrt{3} \times \sqrt{3}) \mathrm{R} 30^{\circ}$ structure has also been reported, resulting in a $(2 \sqrt{3} \times 3)$ rect surface unit cell. ${ }^{56-58}$ It is difficult to determine whether such a modulation occurs in the given case since AFM delivers images averaged over areas well exceeding that of a single molecule; our simulations, however, hint towards a herringbone arrangement as the most stable configuration favoring a $(2 \sqrt{3} \times 3)$ rect unit cell (vide infra).

Independent of the specific structural details, the main conclusion is that both standard and reverse ester SAMs exhibit long-range structural order, with the monolayer structure (especially of the bottom alkyl chain segments) being quite close to that of non-substituted AT SAMs on the same 
substrate. Significantly, the specific geometry of the CmECk molecules defines the orientation of the ester group and, consequently, the direction of the related dipole moment, which is strongly tilted with respect to the surface normal, as shown schematically in Figure 1a. Consequently, its projection on the $z$-axis (i.e., the surface normal), which defines the potential discontinuity within the SAM, depends strongly not only on the ester group orientation but on the exact adsorption geometry of the assembled monolayer. While the average adsorption geometries of the molecules are very similar, one cannot exclude minor variations in different, mid-ester functionalized films discussed in this study (vide infra).
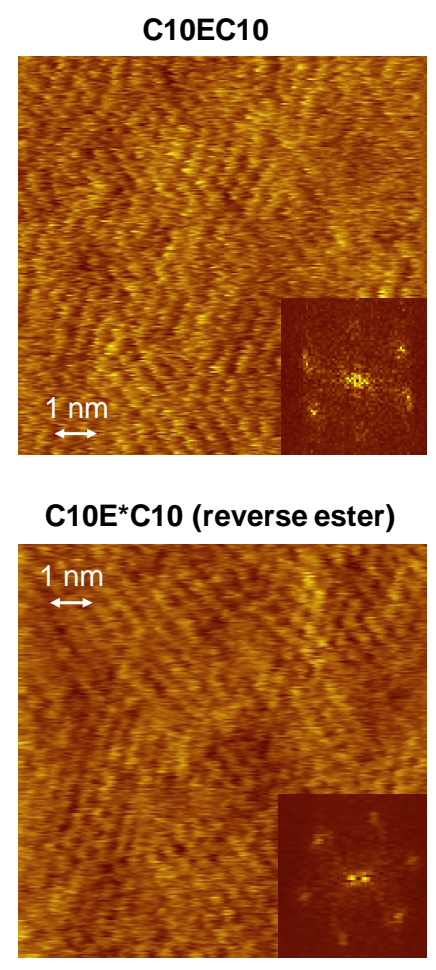

Figure 5. Lateral force AFM topography images of the C10EC10 and C10E*C10 SAMs with 2D Fourier transforms shown in the insets. Both images clearly show a hexagonal pattern which corresponds to the standard $(\sqrt{ } 3 \times \sqrt{ } 3) R 30^{\circ}$ arrangement with a lattice spacing of $\sim 0.50 \mathrm{~nm}$.

\subsection{Electrostatic Effects: Photoemission}

The C 1s XP and HRXP spectra of the Cn SAMs exhibit a single photoemission peak at a BE of 284.85 - $285.0 \mathrm{eV}$ associated with the alkyl backbone. This peak is accompanied by two weak 
shoulders, which strongly overlap with the main peak and can only be recognized within a very detailed analysis. ${ }^{62,63}$ The BE increases somewhat with chain length ( $c a .20 \mathrm{meV}$ per $\mathrm{C}$ atom; see below). ${ }^{62}$ In contrast to these reference systems, the C 1s HRXP spectra of all the CmECk monolayers exhibit not one but two major features related to the alkyl chain, along with two weaker, but easily perceptible peaks assigned to the ether and carbonyl carbons. This pattern is shown in Figure 6, where a spectrum of the C20EC5 SAM serves as a representative example of the mid-chain ester substituted films of this study. Further examples, involving the CmEC5 $(\mathrm{m}=$ 10, 15, and 20) and C10ECk series ( $\mathrm{k}=5,10$, and 15), are presented in the Supporting Information (Figures S3 and S4, respectively). Those spectra exhibit the same pattern as in Figure 6, with the relative intensities of the individual features correlating directly with the lengths of the bottom and top segments, defined by $\mathrm{m}$ and $\mathrm{k}$. The two main contributions to the spectrum at $285.6 \mathrm{eV}$ and $284.75 \mathrm{eV}$ are associated with photoemission peaks from the top and bottom $-\left(\mathrm{CH}_{2}\right)-$ segments, respectively (see color coding in Figure 6). ${ }^{37,38}$ The difference between the BEs of these peaks is $\sim 0.8 \mathrm{eV}$ and all the different embedded ester systems fall within a range of $\pm 0.2 \mathrm{eV}$ of this value. This is shown in the overview summary diagram in Figure 7, presenting the $\mathrm{C} 1 \mathrm{~s}$ BEs associated with the $-\mathrm{CH}_{2}-$ chain or segments for all studied SAMs. This difference cannot be explained within the general concept of chemical shift since both segments are chemically identical. The reason for the difference is, as mentioned above, purely electrostatic, viz. a potential discontinuity inside the monolayer associated with the embedded dipolar ester groups as originally reported in ref 38 and discussed later in detail in ref 37.

Apart from the $\sim 0.8 \mathrm{eV}$ offset, the $\mathrm{C} 1 \mathrm{~s} \mathrm{BEs}$ for the top and bottom $-\mathrm{CH}_{2}-$ segments of the ester SAMs display a similar dependence on the total backbone length as the $\mathrm{C} 1 \mathrm{~s} \mathrm{BE}$ for the $\mathrm{Cn}$ monolayers with a slight increase in the binding energy with increasing backbone length. This dependence stems presumably from a combination of screening and ordering effects, as will be discussed in detail in Section 4.

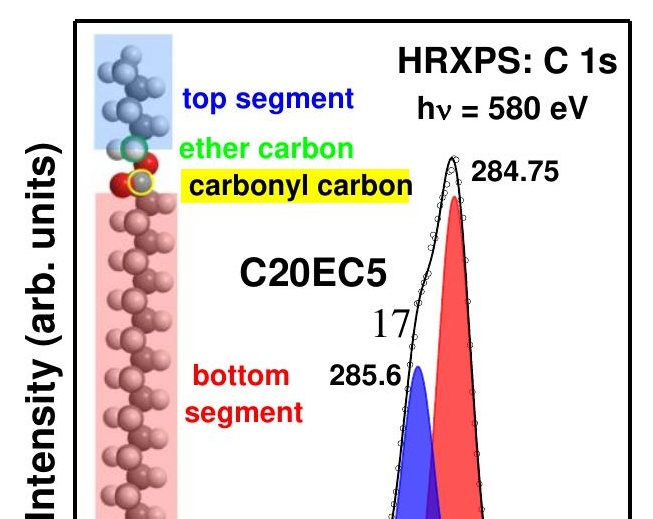


Figure 6. Decomposition of the C 1s HRXP spectrum of the C20EC5 SAM, representative of the entire CmECk series. The individual component peaks associated with specific parts of the C20EC5 molecule are color-coded with the schematics of the molecule shown as an inset. Binding energies of the component peaks are given.

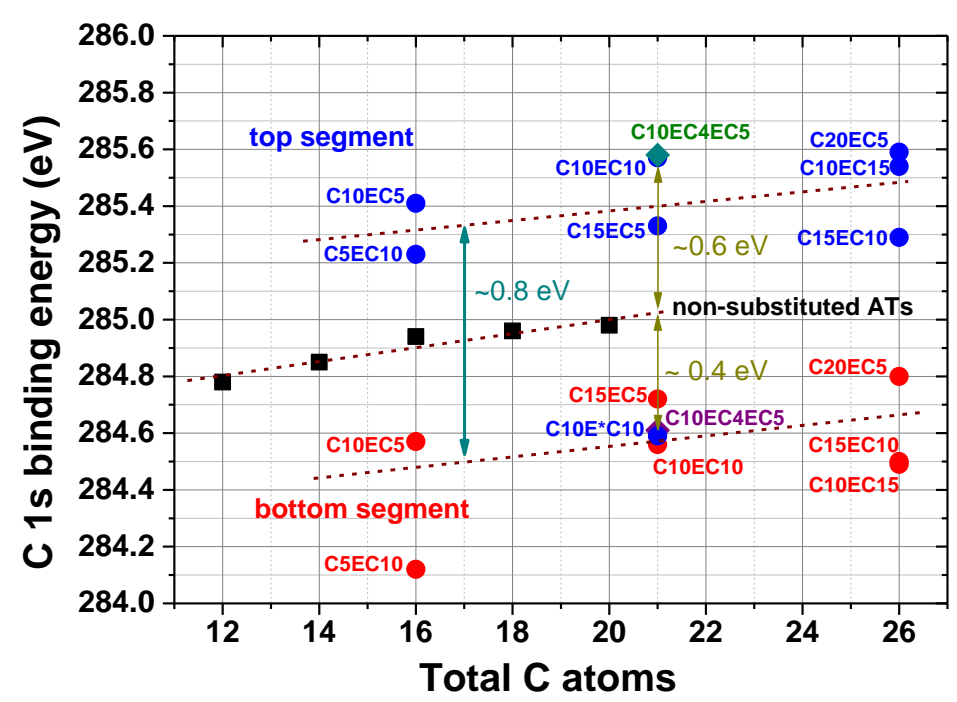

Figure 7. Graphical summary of the $\mathrm{C} 1 \mathrm{~s} \mathrm{BE}$ data for the peak associated with the alkyl chain in the Cn SAMs (black squares) as well as the component peaks related to the top and bottom $-\left(\mathrm{CH}_{2}\right)$ - segments in the mid-ester functionalized AT SAMs: blue and red circles, respectively, for the $\mathrm{CmECk}$ and $\mathrm{C} 10 \mathrm{E}^{*} \mathrm{C} 10 \mathrm{SAMs}$, green and purple rhombus, respectively, for the C10EC4EC5 SAM, for the sake of visibility. The values are the averages over the entire data set (different samples and different photon energies for a particular system). The approximate BE shifts for the top segments in the $\mathrm{C} 10 \mathrm{EC} 10$ and $\mathrm{C} 10 \mathrm{E} * \mathrm{C} 10$ SAMs with respect to the value for the Cn monolayers with a similar number of the carbon atoms in the molecular backbone are given 
and marked by the vertical arrows. The names of the SAMs are given at the respective BEs. The dashed straight lines are guides to the eye.

In addition to analyzing the BE shifts between the top and bottom segments, it is also useful to consider shifts relative to nominally equivalent AT SAMs not bearing any embedded dipolar groups, i.e., to the $\mathrm{Cn}$ series. This is particularly helpful for the reverse ester SAM, C10E*C10, presented also in the overview diagram in Figure 7 and addressed specifically in Figure 8, where the respective $\mathrm{C}$ 1s HRXP spectrum is shown along with the data for the $\mathrm{C} 10 \mathrm{EC} 10$ and $\mathrm{C} 16$ films for the sake of comparison.

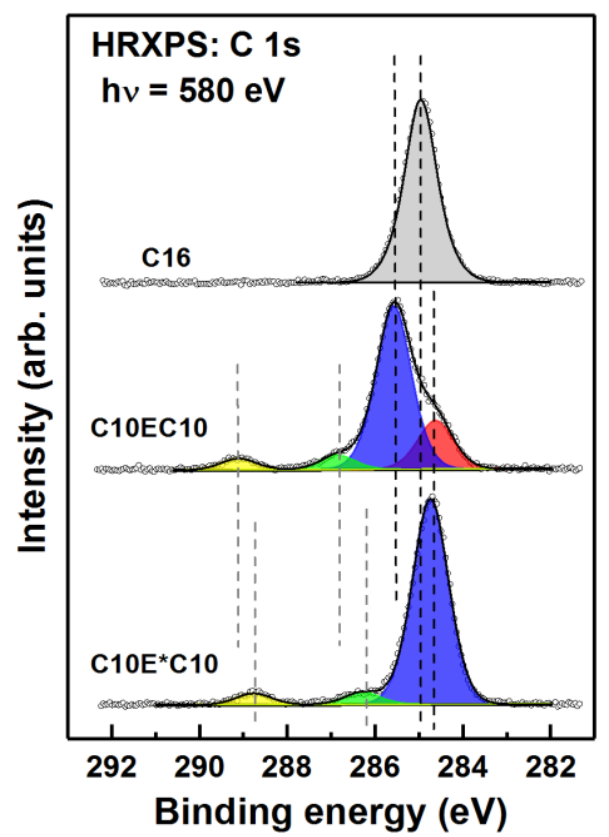

Figure 8. C 1s HRXP spectra of the C16, C10EC10, and C10E*C10 SAMs. The spectra of the two latter films are decomposed into individual component peaks which are color-coded, viz. bottom - $\left(\mathrm{CH}_{2}\right)$ - segment - red, top - $\left(\mathrm{CH}_{2}\right)$ - segment - blue, ether carbon - green, and carbonyl carbon - yellow. The vertical dashed lines mark the BE positions of the component peaks as a guide to the eye.

A careful analysis of this spectrum along with the entire set of the $\mathrm{C} 1 \mathrm{~s}$ data of the involved systems, gives values of +0.6 and $-0.4 \mathrm{eV}$ for the shifts of the main peaks in the $\mathrm{C} 1 \mathrm{~s}$ spectra of 
the $\mathrm{C} 10 \mathrm{EC} 10$ and $\mathrm{C} 10 \mathrm{E}^{*} \mathrm{C} 10$ SAMs compared to the $\mathrm{Cn}$ monolayers with a similar number of the carbon atoms in the molecular chain (see Figure 7; note, this figure contains peak positions averaged over several measurement series, while Figure 8 represents a specific spectrum). The main peaks are exclusively (C10EC10; see above), respectively, predominantly $\left(\mathrm{C}_{10 \mathrm{E}}^{*} \mathrm{C} 10\right.$; see below) associated with the top segment of these SAMs. The different signs of the shifts for the top segment of the $\mathrm{C} 10 \mathrm{EC} 10$ and $\mathrm{C} 10 \mathrm{E}^{*} \mathrm{C} 10$ monolayers compared to the reference $\mathrm{Cn}$ films are fully consistent with inverting the potential step at the ester group. The observation that the BE shift for the ether carbon in $\mathrm{C} 10 \mathrm{E}^{*} \mathrm{C} 10$ is somewhat smaller than the shift of the main peak and the finding that the shift for the carbonyl carbons is smallest also is in line with an inversion of the ester dipole. All mentioned experimental observations are also qualitatively reproduced in the simulations (see section 3.4).

The above discussion relies on associating the main peak of the $\mathrm{C} 10 \mathrm{E}^{*} \mathrm{C} 10 \mathrm{SAM}$ primarily with the $\mathrm{C}$ atoms in the top segment. In contrast to the spectra for regularly oriented esters, for $\mathrm{C} 10 \mathrm{E}^{*} \mathrm{C} 10$, no separate peaks that can be clearly related to the bottom and top segments can be discerned; there is only one intense peak except for the comparably weak ether and carbonyl features. Thus, the main peak in the HRXPS spectrum of this monolayer should be representative of the entire $-\left(\mathrm{CH}_{2}\right)$ - backbone, which means, consequently, that there is no perceptible $\mathrm{C} 1 \mathrm{~s} \mathrm{BE}$ shift between the component peaks associated with the top and bottom segments. The spectral weights of both components in the joint peak should mimic those for the C10EC10 SAM, with the clear dominance of the contribution of the top segment. The BE position of the joint peak, practically coincides with the position of the bottom segment feature in the spectrum of the C10EC10 monolayer, suggesting, thus, that the BE position of the bottom segment feature for the $\mathrm{C} 10 \mathrm{E}^{*} \mathrm{C} 10$ and $\mathrm{C} 10 \mathrm{EC} 10 \mathrm{SAM}$ is non-affected by the orientation of the embedded ester group. Interestingly, this position has a significant BE offset relative to the $\mathrm{Cn} \mathrm{SAMs}$, which is also true for the other CmECk monolayers of this study, as will be discussed in detail in Section 4.

The overview diagram in Figure 7 also contains the results for the double ester SAM, C10EC4EC5. The C 1s HRXP spectrum for this system is presented in Figure 9 which, for the sake of comparison, also contains data for the C10EC10 and C15EC5 monolayers all three of which contain a total of 21 carbon atoms. The $\mathrm{C} 10 \mathrm{EC} 10$ molecule has the same number of $\mathrm{C}$ atoms below and above the first ester group as C10EC4EC5, whereas the same is true for the C15EC5 
compound relative to the second ester group. The spectrum of the C10EC4EC5 SAM is dominated by a broad, asymmetric feature related to the $-\mathrm{CH}_{2}-$ segments and also contains distinct peaks associated with the ether and carbonyl carbons. These ester related peaks are more intense than the analogous peaks in the spectra of the $\mathrm{C} 10 \mathrm{EC} 10$ and C15EC5 monolayers, which is understandable since the C10EC4EC5 SAM contains two ester groups instead of one. As to the major broad feature, its decomposition into individual component peaks can be performed only tentatively, since these peaks are indistinguishable in the overall spectral envelope. Following general logic and based on the data for the CmECk SAMs, three component peaks associated with the bottom, middle, and top $-\mathrm{CH}_{2}-$ segments can be assumed to comprise the joint envelope, with the intensity relations derived from the spectra of the $\mathrm{C} 10 \mathrm{EC} 10$ and $\mathrm{C} 15 \mathrm{EC} 5$ monolayers. The resulting spectral decomposition is presented in Figure 9. While the BE shifts with respect to the component peak assigned to the middle segment can be considered as tentative only, the BE shift between the component peaks associated with the top and bottom $-\mathrm{CH}_{2}-$ segments is presumably more relevant, since it is closely related to the overall spectral shape (it amounts to $\sim 1.25 \mathrm{eV}$ by the three-component-fit and $\sim 0.9 \mathrm{eV}$ when only fitting by two peaks, see Figure S5 in the Supporting Information for the respective decomposition). The value of $1.25 \mathrm{eV}$ for the three-peakdecomposition is somewhat higher than the maximum shift for the CmECk films $(\sim 1.05 \mathrm{eV})$ but noticeably smaller than double the average shift in these films (i.e., $\sim 1.6 \mathrm{eV}$ ). This suggests a distinct but somewhat limited effect of the double embedded ester. An interesting finding in this context is that the particularly clearly resolved feature associated with the carbonyl carbon, which mostly originates from excitations of the top ester group, is hardly shifted compared to the C10EC10 and C15EC5 SAMs. This result suggests that the electrostatic shift caused by the bottom ester group is comparably weak and, concomitantly, the main "electrostatic effect" in this SAM arises from the top ester moiety. 


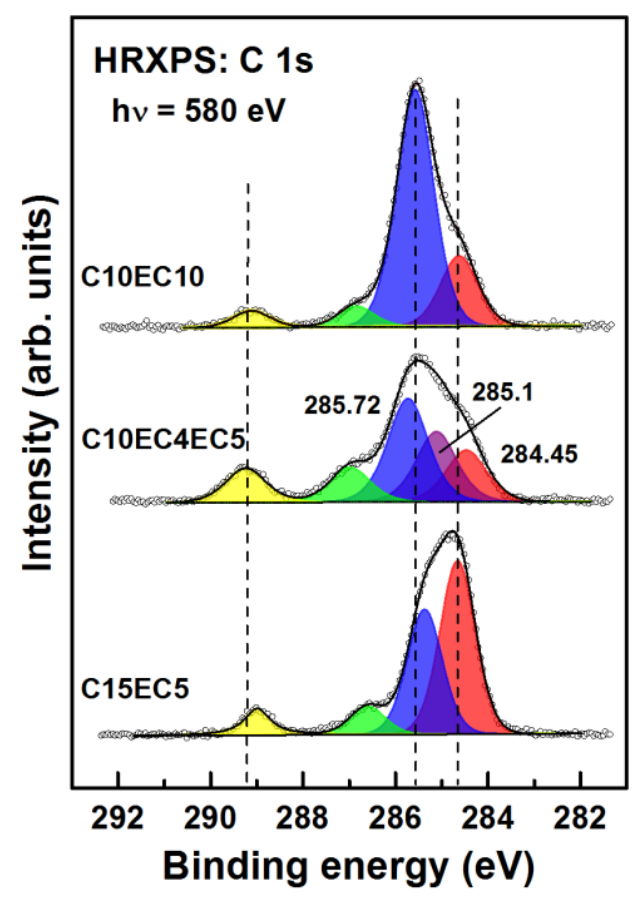

Figure 9. C 1s HRXP spectra of the C10EC10, C10EC4EC5, and C15EC5 SAMs. The spectra are decomposed into individual component peaks that are color-coded, viz. the bottom alkyl segment - red, the middle alkyl segment (for C10EC4EC5 only) - purple, top alkyl segment - blue, ether carbon - green, and carbonyl carbon - yellow. The vertical dashed lines mark the BE positions of the component peaks as a guide to the eye.

\subsection{Electrostatic Effects: Work Function}

The embedding of the dipolar ester group into the alkyl backbone also affects the work function of the SAM-covered Au surface, as shown in Figure 10. That figure contains work function changes, $\Delta \phi$, relative to a $\mathrm{C} 16$ reference SAM, which have been measured by UPS cutoff (for all systems) and by KP-AFM (for the most relevant monolayers). In passing we note that the work function change induced by the C16 SAM relative to a clean Au substrate amounts to $-0.85 \mathrm{eV}$. Also we note that the agreement between the UPS and AFM derived values is good, considering intrinsic differences between these two techniques and the fact that the experiments were independently performed on equivalent, albeit different samples (i.e., silicon versus mica substrates, respectively). This suggests that the samples were homogeneous and not affected in a perceptible way by the character of the substrate within the given choice. 

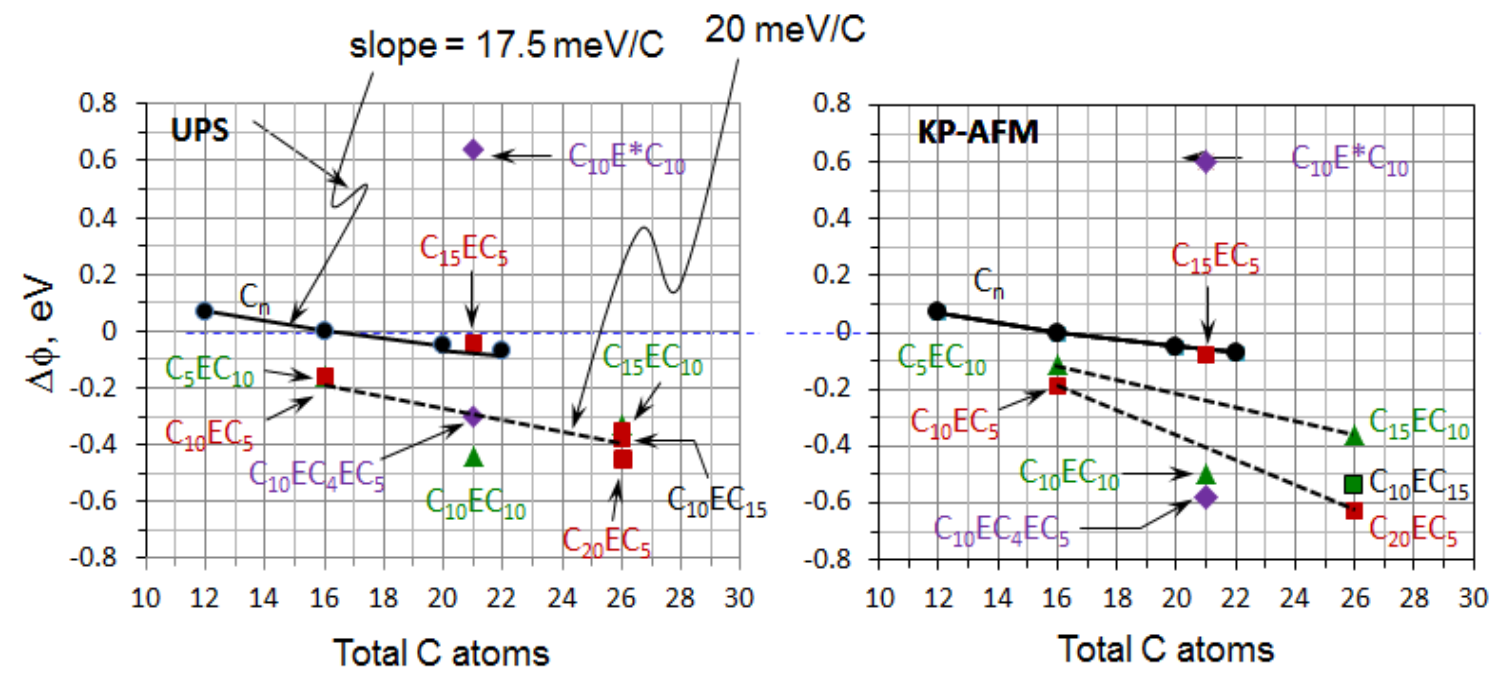

Figure 10. Summary of SAM-induced work function changes relative to a C16 reference SAM. Black circles are the values for the Cn SAMs; all other data points are labelled with the names of the respective ester functionalized monolayers. Left panel: data obtained by the UPS measurements. The slopes of the two main linear correlations are shown by arrows. Right panel: data obtained for selected SAMs by the KP-AFM measurements.

The work function of the Cn SAMs decreases with increasing length of the molecular backbone, which in the past has been explained by a progressive change in the polarizability of the alkyl matrix $^{39,64}$ though it may also be related to improving orientational and conformational order as the chain length increases, as is typical of $\mathrm{Cn}$ monolayers. ${ }^{57} \mathrm{~A}$ similar general trend is observed for the ester-substituted SAMs. In addition, there is a noticeable shift in $\Delta \phi$ between the $\mathrm{Cn}$ and CmECk SAMs, which amounts to -0.20 to $-0.35 \mathrm{eV}$. This shift is similar, albeit slightly smaller, than the shift in core-level BEs between the $\mathrm{Cn}$ and CmECk films (see Figure 7). An exception is the C15EC5 monolayer for which no shift is observed.

Similar to the results of the HRXPS experiments (Figure 7), the reversal of the ester direction leads to a reversal of the sign of $\Delta \phi:-0.40 \mathrm{eV}$ for $\mathrm{C} 10 \mathrm{EC} 10$ and $+0.65 \mathrm{eV}$ for the $\mathrm{C} 10 \mathrm{E} * \mathrm{C} 10 \mathrm{SAM}$ relative to the average value for the C20 and C22 monolayers. This is again fully consistent with the reversal of a potential step inside the SAM that is associated with the vertical component of the ester dipoles. The absolute magnitude of the shift is somewhat larger for the reverse ester SAM, opposite to the observation for the core-level shifts (Figure 7). This difference could be at least in 
part caused by differences in average core-hole screening between the top-segments of the ester containing SAMs and the equally long Cn systems (see Discussion section)

As for the double ester system, the averaged $\Delta \phi$ value for the C10EC4EC5 SAM is comparable to that of the $\mathrm{C} 10 \mathrm{EC} 10$ monolayer (same total number of $\mathrm{C}$ atoms). This result indicates that the effect of the additional embedded ester group on the work function of the entire system is weak. According to the HRXPS data, such a weak effect can potentially be associated with only a minor role played by the bottom ester group, while the top ester provides the major contribution to $\Delta \phi$.

\subsection{Calculated Work Function Modifications and Core-Level Shifts}

\subsubsection{Shift in the Electrostatic Energy and Work Function Change}

The calculated work function changes for $\mathrm{C} 10 \mathrm{EC} 10$ and $\mathrm{C} 10 \mathrm{E}^{*} \mathrm{C} 10 \mathrm{SAMs}$ amount to $-0.62 \mathrm{eV}$ and $+0.88 \mathrm{eV}$, respectively (relative to a herringbone-type C16 SAM; notably, in the simulations we do not observe a pronounced chain-length dependence of the work function of the reference SAMs). These values portray exactly the same trend as the experimental results; only the calculated magnitudes of the $\Delta \phi$ values are larger than those observed in the experiments. This discrepancy is consistent with what has been observed for oligophenylene-based SAMs containing pyrimidine groups at varying orientations. ${ }^{28}$ There the differences were attributed, at least in part, to imperfections in the SAM structure (like the occurrence of grain boundaries). For the embedded ester SAMs, an additional factor is that the calculated tilt-angle of the molecules is $\sim 10^{\circ}$ larger than the experimental one, which results in a larger component of the dipole moment perpendicular to the surface (Figure 1a). 

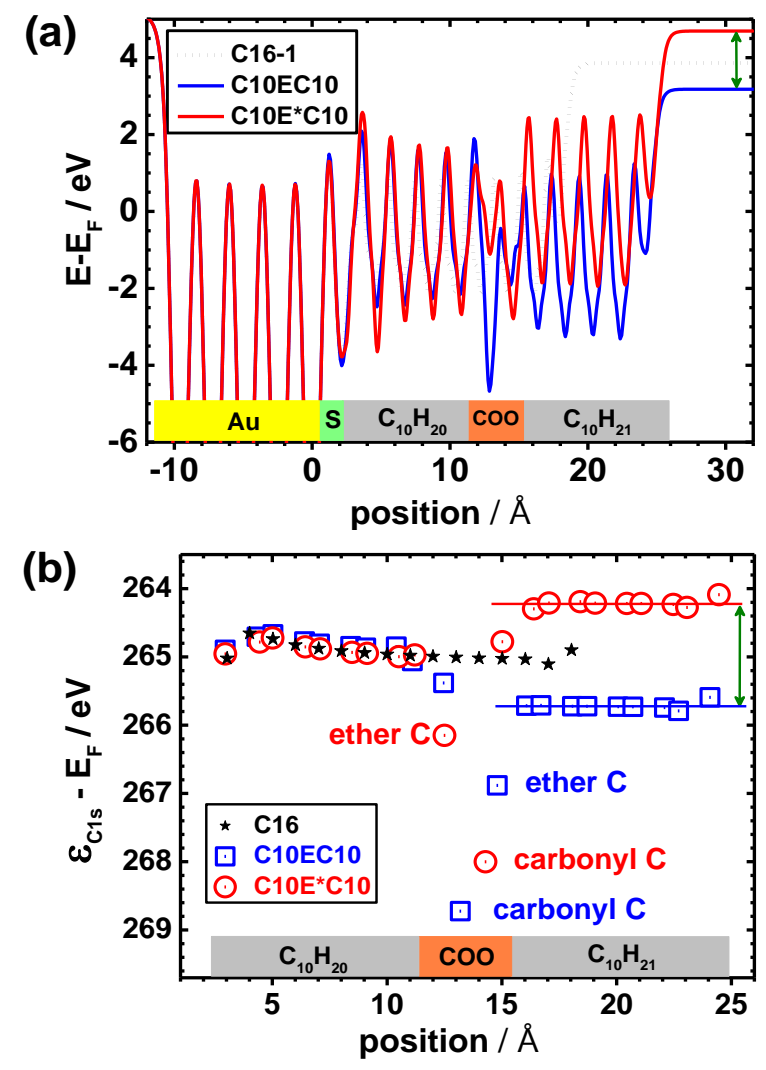

Figure 11. (a) DFT-calculated plane-averaged electrostatic energy of a C16 reference SAM, a $\mathrm{C} 10 \mathrm{EC} 10$ ester SAM, and a $\mathrm{C} 10 \mathrm{E}^{*} \mathrm{C} 10$ reverse ester SAM. The energy is given relative to the Fermi energy. The amplitude of the potential variation differs for the three systems in the SAM region, which is a consequence of not exactly equivalent $\mathrm{C}$ atom positions owing to somewhat different calculated twist angles (note: there are four nominally non-equivalent molecules in the unit cell). (b) Calculated C1s core level energies for the C16, C10EC10 and C10E*C10 SAMs. The zero on the $x$-axis in both plots refers to the average positions of the atoms in the topmost Au layer. The rigid shift between the simulated core-level energies and the experimental BEs is a consequence of the initial state method of calculating core level energies and does not impact the present comparison.

The change in the vacuum energy above the SAM is clearly visible when plotting the electrostatic energy averaged over a plane parallel to the surface of the substrate (see green arrow in the topright corner of Figure 11a). This plot also reveals the origin of the work function change: while the average electrostatic energy between the Au substrate and the ester group is similar for all SAMs, there is a distinct downwards and upwards shift above the ester group, respectively, for the 


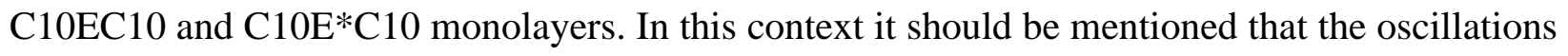
of the plane-averaged electrostatic energy are a consequence of the pronounced minima at the positions of the nuclei. Their contributions to the averaged electrostatic energy sensitively depend on the specific nuclear positions. This can, for example, be seen, when comparing the data for the C16 reference SAM and the ester-containing monolayers, where the different $z$-positions of the nuclei are caused by small variations of the chain twist angles (note that in the calculations each unit cell contains four nominally non-equivalent molecules in a herringbone arrangement). As a consequence, the magnitude of the oscillations provides only limited insight. This becomes apparent, when comparing the data for the C16 SAM in Figure 11a with those for the same monolayer with all chains arranged in parallel (see the Supporting Information).

\subsubsection{Core-Level Excitations}

As discussed in detail in ref 37, the computational approach employed here is able to accurately reproduce the main experimental trends for the HRXP spectra of SAMs in general and the CmECk monolayers in particular. Thus, the calculated core-level energies of a C10EC10, a C10E*C10, and a C16 SAM are plotted in Figure 11b. This plot confirms the assignments of all experimentally observed XPS peaks (vide supra), in particular the association of the two features at $285.5 \mathrm{eV}$ and $284.6 \mathrm{eV}$ with the top and bottom alkyl segments of the C10EC10 SAM. The rigid shift between the simulated and measured core-level energies is a consequence of the initial state method and is not relevant for the present comparison. The BE difference between the top and bottom segments is a direct consequence of collective electrostatic effects arising from the parallel arrangement of the $z$-components of the dipole moments associated with the ester groups. ${ }^{39,40,65}$ This situation results in a step in the electrostatic potential, which shifts the energy of the electronic states above/below that dipole layer relative to each other. Chemically induced shifts of the $\mathrm{C} 1 \mathrm{~s}$ core levels of the ether and carbonyl carbons are superimposed and are also reproduced correctly.

In the modeled, defect-free C10EC5, C5EC10, and C10EC10 SAMs, the shifts between the C 1s core levels for the top and bottom segments are similar and amount to $0.79 \mathrm{eV}$ for C10EC5 and $\mathrm{C} 10 \mathrm{EC} 10$ and $0.85 \mathrm{eV}$ for C5EC10. These values were obtained by averaging over all $\mathrm{C}$ atoms of the respective segments considering changes in the energetic positions of the core-levels due to screening by the metal substrate and weighing the contributions of each core-level considering the damping of the respective signal (for details see the Supporting Information). The calculated shifts 
are quantitatively consistent with the average shifts found experimentally (see Figure 7). The difference between the SAMs containing 5 and 10 carbon atoms in the bottom segment arises in part from differences in core-hole screening for bottom-segment excitations. Additional differences arise from somewhat differing bond-dipoles due to the distinct local bonding geometries associated with odd or even numbers of $\mathrm{m}$ and $\mathrm{k}$ in the CmECk SAMs, as will be discussed below. The impact of electrostatic screening on the overall shift is non-negligible, as, when switching off the mirror-charge screening in the simulations, the BE difference drops to 0.57 $\mathrm{eV}$ for the C5EC10 SAM and to $0.62 \mathrm{eV}$ for C10EC5 monolayer.

When comparing the core-level energies of a regular ester SAM (C10EC10) with a reverse ester one $\left(\mathrm{C}_{10 \mathrm{E}}^{*} \mathrm{C} 10\right)$, we see the main trends of the experiments reproduced in the simulations (Figure 11b): There is a pronounced shift between the top-segment related features of the two SAMs, the overall magnitude of the shift is reduced for the ether carbons, and it is smallest for the carbonyl carbons. The reason for the decreasing shifts is that the corresponding carbons are positioned differently relative to the center of the dipole-induced drop in the electrostatic energy. The overall magnitude of the shift of the XPS peaks/core-level energies for the top segment between C10EC10 and $\mathrm{C}_{10 \mathrm{E}}{ }^{*} \mathrm{C} 10$ are larger in the simulations $(\sim 1.50 \mathrm{eV})$ than in experiments (average value of $\sim 1.0$ $\mathrm{eV}$ ), which is quantitatively consistent with what has been observed for the work function changes (vide supra).

What the simulations presented so far cannot answer directly, are some conundrums mentioned when discussing the experimental spectra: why are the core-level BEs in the C16 reference SAMs profoundly shifted relative to those of the bottom segments of the ester-containing monolayers? Referring to Figure 8, why there is no perceptible shift in the BE position of the C 1s peak associated with the top segments of the $\mathrm{C} 10 \mathrm{E}^{*} \mathrm{C} 10 \mathrm{SAM}$ as compared to the bottom segments one? Why does the C15EC5 SAM yield a work function similar to the non-substituted AT SAMs? In the following Section, based on carefully designed test calculations, we hypothesize that these aspects are at least in part related to structural imperfections within the films.

\subsubsection{Impact of Possible Structural Imperfections}


A particularly relevant imperfection arises from chains in which the twist angle is changed by $180^{\circ}$, as shown in the inset of Figure 12. In such a configuration the molecules do not need more space or significantly disturb their neighbors. Thus, the twisting is expected to not significantly affect the main structural properties of the SAM, like the measured tilt and the orientation of the plane of the carbon atoms, i.e., it might go unnoticed even for films characterized as thoroughly as the present ones.

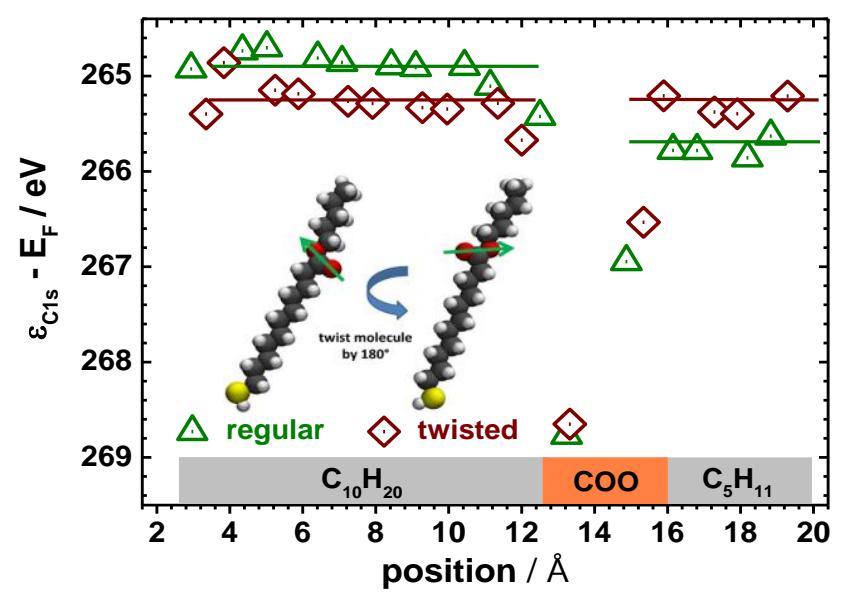

Figure 12. DFT-calculated C 1s core level energies for the C10EC5 SAMs in the regular (green) and twisted (brown) configurations. The zero on the $x$-axis in both plots refers to the average positions of the atoms in the topmost Au layer.

In spite of the structural consequences of the twist-defect being only minor; the reorientation of the ester group drastically impacts the electronic properties of the SAM. This is evidenced by BE differences and work function changes for various chain imperfections (see the Supporting Information). For example, the C 1s BEs of the C10EC5 SAM shown in Figure 12 demonstrate the two main effects of the twisting imperfections: (i) There is no longer a significant shift in the core-level energies between the top and bottom segments, as the orientation of the ester dipole in the twisted SAM is essentially horizontal; (ii) the modified bonding geometry in the region of the thiolate group changes the dipole jump at the interface with the Au substrate (i.e., the sulfur-gold bond dipole), which results in a rigid shift of all core levels. The magnitude of the two effects varies between different SAMs. For example, while the rigid potential shift at the SAM/Au interface (measured as the difference in core level energies between the bottom segment and a C16 
$\mathrm{SAM}$ ) in the twisted configuration of C10EC5 amounts to $+0.35 \mathrm{eV}$, it is only $+0.22 \mathrm{eV}$ for $\mathrm{C} 10 \mathrm{E}^{*} \mathrm{C} 10$ and becomes negligibly small for C5EC10.

It is also noteworthy that for the latter SAM, due to the odd number of $\mathrm{C}$ atoms in the bottom segment, a chain configuration at the thiolate equivalent to that shown in Figure 1 would result in an essentially horizontal dipole moment of the ester group, which would eliminate the potential step. Thus, to provide an appreciable potential step in C5EC10, the local bonding geometry in the vicinity of the thiolate must correspond to that of the "twisted" rather than the "regular" configuration (see the sketch for C10EC5 in Figure 12).

The possible occurrence of twist-defects severely complicates the situation. For example, a change of the dipole in the thiolate region $\left(\mu_{\text {thio }}\right)$ rigidly shifts the core-level energies of both the top as well as the bottom segments and it also impacts the work function modification. Still, it does not directly impact the core-level shift between the top and bottom segments. Conversely, the latter quantity and the work function modification are impacted by the orientation of the ester-dipole, which, however, has no consequences for the core-level binding energies of the bottom segment (recall that the all BEs are all referenced to the gold substrate which is located under the dipole layer as is the bottom segment). It should also be noted that beyond twisted chains, reconstructions

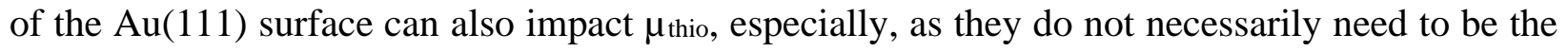
same for non-substituted ATs and substituted analogues. ${ }^{40,66}$ Finally, a very significant point to keep in mind is that core-level shifts are mostly impacted by "the local electrostatic environment" of the carbon atom from which the electron is excited, while work function changes "average" over much larger areas. As a consequence, for films with scattered defects or defective regions, both quantities react differently depending on whether imperfectly aligned chains are phase separated or distributed homogeneously within the film. ${ }^{29}$

An important issue in this context is, under which circumstances regular and twisted configurations are stabilized. To address that, we performed molecular dynamics simulations on extended test systems (comprising unit cells containing 288 molecules), where we observe a bimodal distribution of the ester dipoles corresponding to the aforementioned regular and twisted arrangements. In those simulations, ${ }^{67}$ we found a preferentially regular arrangement for C10EC5 SAMs, a non-negligible contribution of twisted chains for $\mathrm{C} 10 \mathrm{EC} 10$ and even a dominance of twisted chains for C5EC10 (owing to the odd number of the carbon atoms in the bottom segment, 
as discussed above). Interestingly, those twisted structures are randomly distributed amongst properly aligned chains rather than precipitating into separate phases. ${ }^{67}$ These results do not necessarily reflect the experimental reality owing to the use of empirical force-fields parameterized for systems other than thiolate SAMs (see the Supporting Information). Still, they at least hint towards the possible existence of twisted phases. In fact, for C5EC10, even the DFT-based simulations indicate that the twisted phase is more stable than the regular one by $0.075 \mathrm{eV}$ per molecule.

In the experiments, there is no definite indication for a massive occurrence of molecules in a twisted configuration in any of the studied SAMs. This conclusion is evidenced by the similar intensity values of the $\mathrm{C}=\mathrm{O}$ stretching mode peaks in Figure 2a,c since the horizontal orientation of the ester group would shut off the appearance of this mode, thus providing intensity patterns not consistent with those in the figure. Nevertheless, it is intriguing that the molecules with an odd number of carbon atoms in the bottom segment consistently have a lower $\mathrm{C} 1 \mathrm{~s}$ binding energy for the upper segment than those with an even number of carbons. Chain twisting might also play a role for the vanishing $\Delta \phi$ for the C15EC5 monolayer compared to an equivalently long Cn SAM.

\section{Discussion}

All mid-chain ester functionalized CmECk SAMs studied here exhibit pronounced electrostatic effects arising from the collective superposition of the fields associated with the embedded dipole group. This is in agreement with previous studies on some of these systems ${ }^{37,38}$ as well as on analogous thioaromatic monolayers with embedded pyrimidine groups. ${ }^{28,29}$ The electrostatic effects are manifested in two ways: as a BE shift of the $\mathrm{C} 1 \mathrm{~s}$ peaks associated with the bottom and top $-\mathrm{CH}_{2}-$ segments of the molecular backbone and as a change in the work function of the entire system. The former implies that the general concept of a mere chemical shift of XPS features explains only part of the effects encountered in ordered polar assemblies. Rather, electrostatically induced shifts of the energy landscape also need to be considered on an equal footing. ${ }^{37}$ This behavior is in some sense reminiscent of the role of the Madelung potential for XPS spectra of ionic crystals. ${ }^{68-70}$

Correspondingly, the $\mathrm{C}$ 1s core-level energies in the regions above and below the dipoles differ relative to one another in the experiments for most of the CmECk SAMs by $\sim 0.8 \mathrm{eV}$ (see Figure 
7). This result is fully consistent with all our DFT calculations on extended interfaces and also correlates nicely with a simple theoretical estimate based on the orientation of the ester groups in the SAMs and their dipole moment. ${ }^{38} \mathrm{~A}$ minor variation in the differences in BEs $( \pm 0.2 \mathrm{eV})$ can be tentatively explained by the occurrence of structural imperfections. Since the dipole associated with the embedded ester group is strongly tilted with respect to the surface normal, even small structural changes can result in a noticeable change of its component perpendicular to the surface. Moreover, as discussed in section 3.4.3, in our simulations we observed a low-energy conformation with essentially horizontal dipole orientations.

Another relevant aspect is that screening-induced shifts of BEs depend on the position of the corehole relative to the metal substrate and the SAM/ambient interface. The impact of core-hole screening by the metal can be estimated from the simple mirror-charge model described in the Supporting Information. Within this model (considering the finite escape depth of the photoelectrons but neglecting the impact of the finite thickness of the SAMs), screening effects decrease the averaged BE of the top segment of C10EC5 by $0.10 \mathrm{eV}$ and of C10EC10 by $0.08 \mathrm{eV}$. The effect is naturally much stronger for the bottom segments and amounts to $0.26 \mathrm{eV}$ in both cases; i.e., the core-hole screening contributes $\sim 0.17 \mathrm{eV}$ (C10EC5) and $\sim 0.18 \mathrm{eV}$ (C10EC10) to the splitting between the peaks associated with the top and bottom segments. Also for the reference SAMs, where the net screening is intermediate, a decrease in the screening efficiency with increasing chain length can be expected, which is at least one of the factors causing the measured $\mathrm{BE}$ shifts as a function of chain length shown in Figure 7. A particularly strong impact of the screening asymmetry on BEs can be expected for systems with short bottom segments, such as C5EC10. Indeed, there, the screening-induced shift of the average bottom segments related BE is $0.38 \mathrm{eV}$. This shift results in an even larger contribution of screening to the BE difference between the top and bottom segments.

As mentioned in Section 3.2, in addition to analyzing the shifts in BEs between the top and bottom segments, it is also useful to consider shifts relative to nominally equivalent non-substituted AT SAMs (i.e., the Cn series). Surprisingly, there is a rigid shift of $\sim 0.4 \mathrm{eV}$ in the BEs of the chemically essentially identical bottom segments of the CmECk SAMs and the equally long $\mathrm{Cn}$ SAMs (with $n=m+k$ ). To analyze the origin of that shift, it is instructive to replot the data as a function of the number of bottom segment $\mathrm{C}$ atoms (done in Figure 13) rather than as a function 
of the total molecular length (Figure 7). The motivation for that is that the length of the bottom segment is what primarily determines the impact of core-hole screening on the peak position (vide supra). Comparing the data in Figure 7 with those in Figure 13 one sees that the shift is, indeed, reduced to $\sim 0.25 \mathrm{eV}$, i.e., more than a third of the shift in the peak positions can be associated with substrate-related screening differences. The reason for the remaining BE lowering in the estercontaining SAMs is not yet understood. We hypothesize that it might be a consequence of differences in the bonding- and thiolate-related dipoles at the metal/SAM interface. These could be caused by differences in bonding geometries, substrate reconstructions, or structural imperfections in general (see Section 3.4.3).

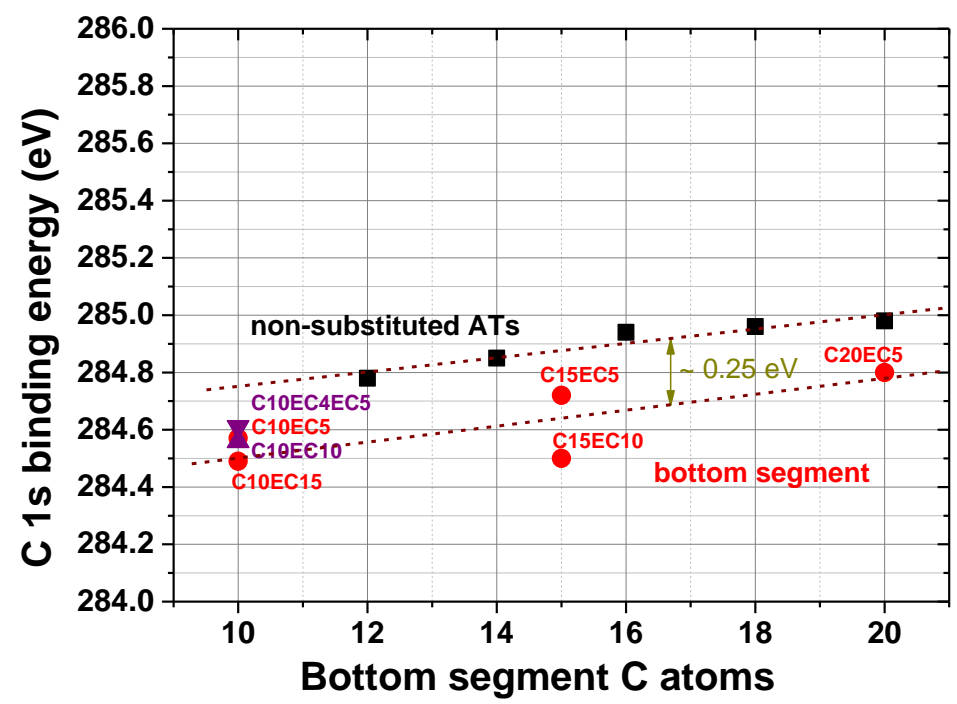

Figure 13. Graphical summary of the $C 1 \mathrm{~s} \mathrm{BE}$ data for the peak associated with the alkyl chain in the $\mathrm{Cn}$ SAMs (black squares) as well as the component peak related to the bottom - $\left(\mathrm{CH}_{2}\right)$ - segment in the mid-ester functionalized AT SAMs (red circles; only C10EC10 and C10EC4EC5 are marked by purple up and down triangles for the sake of visibility). The values are the averages over the entire data set (different samples and different photon energies for each system). The C 1s BE values are arranged in accordance with the length of the bottom segment. The approximate energy difference between the average values for the $\mathrm{Cn}$ and CmECk SAMs is indicated by the vertical arrow. The names of the SAMs are given at the respective BEs. The brown dashed straight lines are guides to the eye.

The use of the Cn SAMs as a reference also results in a smaller value of the electrostatic shift associated with the top segment in the ester SAMs, viz. +(0.3-0.55) eV. These values are in good agreement with the work function changes induced by the CmECk SAMs measured again relative 
to $\mathrm{Cn}$ reference samples of equivalent lengths (between $-0.2 \mathrm{eV}$ and $-0.35 \mathrm{eV}$; see Figure 10). Notably larger shifts of up to $-0.6 \mathrm{eV}$ are obtained when compared to a single reference SAM (namely C16, as it is done in Figure 10). The reason for that difference is that the work function change induced by the CmECk SAMs follows a roughly linear evolution with the total number of $\mathrm{C}$ atoms (Figure 10). This mimics the behavior of the $\mathrm{Cn}$ films with a slope of $-17.5 \mathrm{meV}$ per $\mathrm{C}$ atom in the backbone.

An interesting observation regarding the variation between the different systems beyond the aforementioned effects is that the CmECk SAMs with an odd number of carbon atoms in the bottom segment display smaller shifts in the BEs of the top segments (see Figure 7) and they also induce smaller work function changes (Figure 10). An explanation for that could be that these SAMs are more prone to SAM imperfections comprising twisted configurations with essentially horizontally oriented ester dipoles (see Section 3.4.3). What is still somewhat puzzling is the observation that $\Delta \phi$ for the C15EC5 SAM is close to zero relative to C20, although the experimentally accessible structural parameters of that film are similar to those of the other samples. A higher concentration of twisted conformations in C15EC5 is a viable scenario, supported by the comparably small shift in BEs between the top and bottom segments. Still that shift amounts to $0.61 \mathrm{eV}$, which appears too large to be merely-screening induced.

At this point, it should also be mentioned that the average orientation of the terminal methyl groups (carrying a non-vanishing dipole moment ${ }^{71}$ ) could affect work function changes, but would have only a minor impact on BE shifts. Changes in the orientation of the $-\mathrm{CH}_{3}$ segments are either simply a consequence of differences in chain length (so called odd-even effects) or can be caused by different degrees of the conformational and orientational disorder in the top segments of the SAMs. To address the role of the odd-even effects, we calculated work function changes of nonsubstituted AT SAMs of varying chain lengths. There, we did indeed observe systematic variations, but their magnitude was comparably small, amounting to only $0.05 \mathrm{eV}$ for work function differences between chains containing odd, respectively even numbers of $\mathrm{C}$ atoms.

As far as the "double ester" SAM, C10EC4EC5, is concerned, one might expect that this layer would induce shifts in work function and BE differences that are roughly twice as large as for SAMs consisting of molecules containing only a single ester group. This is, however, not what we observe. Rather, the value of $\Delta \phi$ for that SAM is comparable to that for the similarly long $\mathrm{C} 10 \mathrm{EC} 10$ 
SAM. Moreover, even though a unambiguous decomposition of the HRXP spectrum of C10EC4EC5 is not possible (see Figure 9 and Figure S5 in the Supporting Information), the BE difference between the $\mathrm{C}$ 1s peaks associated with the bottom and top $-\mathrm{CH}_{2}-$ segments (limited by the spectral envelope) is relatively close to that of the C10EC10 SAM. All these aspects point towards a reduced electrostatic impact of at least one of the two embedded dipoles (very likely the bottom one as gauged from the very small shift of the carbonyl carbon related peak (vide supra). This stems presumably from its specific orientation. A potentially higher degree of disorder in the C10EC4EC5 system could well be a consequence of the fact that in a double ester SAM we deal with the molecules with two kinks along their backbones. This implies that the average tilt angles of the individual segments have to increasingly deviate from the ideal values of $\mathrm{Cn}$ and $\mathrm{CmECk}$ SAMs.

While the C10EC4EC5 SAMs do not exhibit their full ideal potential contributions from both ester dipoles, the "reverse ester", $\mathrm{C} 10 \mathrm{E}^{*} \mathrm{C} 10$, film displays very significant and interesting properties. With respect to the $\mathrm{Cn}$ reference, the reversal of the direction of the embedded dipole results in an average BE shift of the top segment of ca. $-0.4 \mathrm{eV}$, in contrast to the value of $+0.6 \mathrm{eV}$ for the C10EC10 monolayer. Such an inversion of the BE shift is consistent with the reversal of the vertical direction of the ester-dipole. It also matches the observed shifts of the BEs of the ether and carbonyl carbons (Figure 8). Still, it should also be mentioned that we do not fully understand,

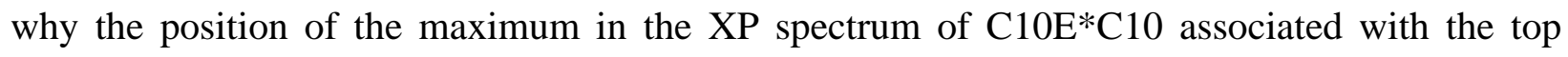
segment is so close to that associated with bottom segment of C10EC10.

Finally, from a practical point of view, it is important to point out that all work function changes discussed above are not accompanied by a significant change in molecular orientation and SAM structure, as follows from the IRS (Figures 2-4), NEXAFS spectroscopy, ${ }^{38}$ and AFM (Figure 5) data. Moreover, the dipole control is fully decoupled from the interfacial chemistry, which makes such SAMs very useful tools for the molecular engineering of interfaces, an aspect we recently also exploited for embedded-dipole based aromatic monolayers. ${ }^{28}$

\section{Conclusions and Prospects}

We have shown that AT SAMs containing embedded dipolar ester groups are highly attractive materials for modifying the properties of metal substrates. They form well-defined and highly ordered layers and enable modifying the substrate work function in a range of $\pm 0.6 \mathrm{eV}$ compared 
to a non-substituted analogue. The sign of the work function change depends on the orientation of the embedded ester group and, most importantly, the change is achieved without affecting the SAM-ambient interface. Incorporating two ester groups into the backbone did not increase the overall effect noticeably for a test system of this study but is still probably possible at a favorable orientation of both these groups.

A thorough investigation of nine embedded ester systems also provides insight into the electronic structure within the SAMs: combining high-resolution XPS data with state-of-the-art slab-type band-structure calculations, we find a step in the electrostatic energy at the position of the embedded ester groups, which is caused by the collective interaction of the molecular dipoles. This effect shifts the core-level energies of otherwise chemically equivalent carbon atoms that are below and above the esters relative to each other. Moreover, a shift of the $\mathrm{C} 1 \mathrm{~s}$ BEs of both the top as well as the bottom segments of the SAM relative to non-substituted ATs of equivalent length is also observed. Our results suggest that the magnitude of the work function change as well as the core-level shifts weakly increase (approximately linearly) with chain length, which we tentatively associate with an increased ordering of the longer chains. As far as the XPS data are concerned, decreasing average core-hole screening for longer chains also plays a role.

As evidenced by comparing the various systems, the magnitude of the core-level and work function shifts sensitively depends on the details of the film structure, which is not surprising considering the strongly inclined orientation of the ester dipoles relative to the surface normal. The simulations also imply that the occurrence of alkanethioleate chains, whose twist angle is modified by $180^{\circ}$ is not an unlikely scenario. While those chains are not expected to affect the structural order of the layers significantly and are difficult to trace spectroscopically, the twisting results in a nearhorizontal alignment of the ester dipoles. The concurrent change of the bonding geometry at the thiolate group also modifies the respective bond dipole. In other systems with chemical functionality built into the molecular backbone, we have previously found significant effects due to networks of molecules interacting underneath the SAM surface. ${ }^{72}$ Overall, the investigated systems display a variety of interesting properties, whose detailed analysis is intricate and requires the combination of a variety of complementary techniques. A particular experimental tool, which can be relevant for these systems, is second harmonic generation spectroscopy, that can provide information on the orientation of the terminal methylene groups in the ester SAMs, important in context of the work function. 


\section{Associated Content}

Supporting Information. Details of the surface potential measurements; additional HRXPS data; additional details on the band-structure calculations; additional details on molecular dynamics modeling; plane averaged electrostatic energies - geometry dependence of energy-oscillations; and summary of calculated core-level shifts and work function changes. This information is available free of charge via the Internet at http://pubs.acs.org.

\section{- Author Information}

\section{Corresponding Authors}

*(M.Z.) E-mail: Michael.Zharnikov@urz.uni-heidelberg.de;

*(E.Z.) E-mail: egbert.zojer@tugraz.at;

*(D.L.A.) E-mail: dla3@psu.edu

\section{Present Addresses}

a(O.M.C.) Bausch \& Lomb, Rochester, NY, United States

b(S.S.) HOFFMANN EITLE Patent- und Rechtsanwälte PartmbB, München, Germany

c(P.P.Z.) Dept. of Physics, Michigan State University, Lansing, MI, United States

d(M.M.M.) Research Center for Adv. Sci. and Tech., The University of Tokyo, Tokyo, Japan

e(N.S.) Intelligent Fusion Technology, Germantown, MD 20876, United States

\section{Notes}

${ }^{\#}$ O.M.C., S.S., and I.H. contributed equally to the given work.

The authors declare no competing financial interest.

\section{- Acknowledgments}

The authors thank the Max IV lab for the allocation of the beamtime and Max IV staff, including A. Preobrajenski in particular, for the technical support. We also acknowledge the help of M. Vieider, O. M. Roscioni, L. Muccioli, and C. Zannoni with performing the MD simulations. This 
work has been supported financially by the German Research Foundation (DFG) (grants ZH 63/171 and ZH 63/22-1), by the Austrian Science Fund (FWF): P24666-N20, I2081-N20, and I937-N19, by the US Army Research Office (DA, OC), the US National Science Foundation (NS), and the US Department of Energy, grant \#DE-SC-1037004 (PSW). The computational results presented have been achieved mostly using the Vienna Scientific Cluster (VSC). 


\section{- References}

(1) Ulman, A. Formation and Structure of Self-Assembled Monolayers. Chem. Rev. 1996, 96, 1533-1554.

(2) Schreiber, F. Self-Assembled Monolayers: From Simple Model Systems to Biofunctionalized Interfaces. J. Phys. Condens. Matter 2004, 16, R881-R900.

(3) Love, J. C.; Estroff, L. A.; Kriebel, J. K.; Nuzzo, R. G.; Whitesides, G. M. SelfAssembled Monolayers of Thiolates on Metals as a Form of Nanotechnology. Chem. Rev. 2005, 105, 1103-1169.

(4) Campbell, I. H.; Rubin, S.; Zawodzinski, T. A.; Kress, J. D.; Martin, R. L.; Smith, D. L.; Barashkov, N. N.; Ferraris, J. P. Controlling Schottky Energy Barriers in Organic Electronic Devices Using Self-Assembled Monolayers. Phys. Rev. B 1996, 54, 14321-14324.

(5) Campbell, I. H.; Kress, J. D.; Martin, R. L.; Smith, D. L.; Barashkov, N. N.; Ferraris, J. P. Controlling Charge Injection in Organic Electronic Devices Using Self-Assembled Monolayers. Appl. Phys. Lett. 1997, 71, 3528-3530.

(6) Salinas, M.; Jäger, C. M.; Amin, A. Y.; Dral, P. O.; Meyer-Friedrichsen, T.; Hirsch, A.; Clark, T.; Halik, M. The Relationship between Threshold Voltage and Dipolar Character of SelfAssembled Monolayers in Organic Thin-Film Transistors. J. Am. Chem. Soc. 2012, 134, 1264812652.

(7) Crivillers, N.; Osella, S.; Van Dyck, C.; Lazzerini, G. M.; Cornil, D.; Liscio, A.; Di Stasio, F.; Mian, S.; Fenwick, O.; Reinders, F.; et al. Large Work Function Shift of Gold Induced by a Novel Perfluorinated Azobenzene-Based Self-Assembled Monolayer. Adv. Mater. 2013, 25, 432-436.

(8) Lange, I.; Reiter, S.; Pätzel, M.; Zykov, A.; Nefedov, A.; Hildebrandt, J.; Hecht, S.;

Kowarik, S.; Wöll, C.; Heimel, G.; et al. Tuning the Work Function of Polar Zinc Oxide Surfaces using Modified Phosphonic Acid Self-Assembled Monolayers. Adv. Funct. Mater. 2014, 24, 7014-7024.

(9) Kim, J.; Rim, Y. S.; Liu, Y.; Serino, A. C.; Thomas, J. C.; Chen, H.; Yang, Y.; Weiss, P. S. Interface Control in Organic Electronics Using Mixed Monolayers of Carboranethiol Isomers. Nano Lett. 2014, 14, 2946-2951. 
(10) Margapoti, E.; Li, J.; Ceylan, Ö.; Seifert, M.; Nisic, F.; Le Anh, T.; Meggendorfer, F.; Dragonetti, C.; Palma, C.-A.; Barth, J. V.; et al. A 2D Semiconductor-Self-Assembled Monolayer Photoswitchable Diode. Adv. Mater. 2015, 27, 1426-1431.

(11) Bock, C.; Pham, D. V.; Kunze, U.; Kaefer, D.; Witte, G.; Woell, C. Improved Morphology and Charge Carrier Injection in Pentacene Field-Effect Transistors with ThiolTreated Electrodes. J. Appl. Phys. 2006, 100, 114517.

(12) Ford, W. E.; Gao, D.; Knorr, N.; Wirtz, R.; Scholz, F.; Karipidou, Z.; Ogasawara, K.; Rosselli, S.; Rodin, V.; Nelles, G.; et al. Organic Dipole Layers for Ultralow Work Function Electrodes. ACS Nano 2014, 8, 9173-9180.

(13) Gartsman, K.; Cahen, D.; Kadyshevitch, A.; Libman, J.; Moav, T.; Naaman, R.; Shanzer, A.; Umanshy, V.; Vilan, A. Molecular Control of a GaAs Transistor. Chem. Phys. Lett. 1998, 283, 301-306.

(14) Cohen, R.; Kronik, L.; Shanzer, A.; Cahen, D.; Liu, A.; Rosenwaks, Y.; Lorenz, J. K.; Ellis, A. B. Molecular Control over Semiconductor Surface Electronic Properties: Dicarboxylic Acids on CdTe, CdSe, GaAs, and InP. J. Am. Chem. Soc. 1999, 121, 10545-10553.

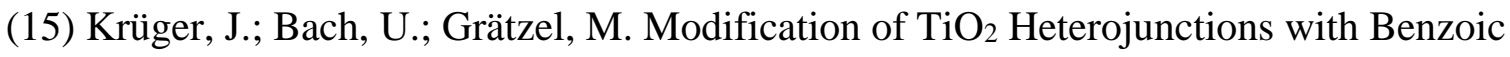
Acid Derivatives in Hybrid Molecular Solid-State Devices. Adv. Mater. 2000, 12, 447-451.

(16) Ashkenasy, G.; Cahen, D.; Cohen, R.; Shanzer. A.; Vilan. A. Molecular Engineering of Semiconductor Surfaces and Devices. Acc. Chem. Res. 2002, 35, 121-128.

(17) Wu, D. G.; Cahen, D.; Graf, P.; Naaman, R.; Nitzan, A.; Shvarts, D. Direct Detection of Low-Concentration NO in Physiological Solutions by a New GaAs-Based Sensor. Chem. Eur. J. 2001, 7, 1743-1749.

(18) Takeya, J.; Nishikawa, T.; Takenobu, T.; Kobayashi, S.; Iwasa, Y.; Mitani, T.; Goldman, C.; Krellner, C.; Batlogg, B. Effects of Polarized Organosilane Self-Assembled Monolayers on Organic Single-Crystal Field-Effect Transistors. Appl. Phys. Lett. 2004, 85, 5078-5080.

(19) Kim, J.; Rim, Y. S.; Chen, H.; Cao, H. H.; Nakatsuka, N.; Hinton, H. L.; Zhao, C.; Andrews, A. M.; Yang, Y.; Weiss, P. S. Fabrication of High-Performance Ultrathin $\mathrm{In}_{2} \mathrm{O}_{3}$ Film Field-Effect Transistors and Biosensors Using Chemical Lift-Off Lithography. ACS Nano 2015, 9, 4572-4582. 
(20) Cahen, D. Kahn, A. Electron Energetics at Surfaces and Interfaces: Concepts and Experiments. Adv. Mater. 2003, 15, 271-277.

(21) Alloway, D. M.; Hofmann, M.; Smith, D. L.; Gruhn, N. E.; Graham, A. L.; Colorado, Jr., R.; Wysocki, V. H.; Lee, T. R.; Lee, P. A.; Armstrong, N. R. Interface Dipoles Arising from Self-Assembled Monolayers on Gold: UV-Photoemission Studies of Alkanethiols and Partially Fluorinated Alkanethiols. J. Phys. Chem. B 2003, 107, 11690-11699.

(22) Palermo V.; Palma, M.; Samorì, P. Electronic Characterization of Organic Thin Films by Kelvin Probe Force Microscopy. Adv. Mater. 2006, 18, 145-164.

(23) Hamadani, B. H.; Corley, D. A.; Ciszek, J. W.; Tour, J. M.; Natelson, D. Controlling Charge Injection in Organic Field-Effect Transistors Using Self-Assembled Monolayers. Nano Lett. 2006, 6, 1303-1306.

(24) Heimel, G.; Romaner, L.; Brédas, J. L.; Zojer, E. Interface Energetics and Level Alignment at Covalent Metal-Molecule Junctions: p-conjugated thiols on gold, Phys. Rev. Lett. 2006, 96, 196806.

(25) Schmidt, C.; Witt, A.; Witte, G. Tailoring the $\mathrm{Cu}(100)$ Work Function by Substituted Benzenethiolate Self-Assembled Monolayers. J. Phys. Chem. A 2011, 115, 7234-7241.

(26) Hohman, J. N.; Zhang, P.; Morin, E. I.; Han, P.; Kim, M.; Kurland, A. R.; McClanahan, P. D.; Balema, V. P.; Weiss, P. S. Self-Assembly of Carboranethiol Isomers on Au\{111\}: Intermolecular Interactions Determined by Molecular Dipole Orientations. ACS Nano 2009, 3, 527-536.

(27) Yoon, H. J.; Bowers, C. M.; Baghbanzadeh, M.; Whitesides, G. M. The Rate of Charge Tunneling Is Insensitive to Polar Terminal Groups in Self-Assembled Monolayers in $\operatorname{AgTSS}\left(\mathrm{CH}_{2}\right)_{\mathrm{n}} \mathrm{M}\left(\mathrm{CH}_{2}\right)_{\mathrm{m}} \mathrm{T} / \mathrm{Ga}_{2} \mathrm{O}_{3} / \mathrm{EGaIn}$ Junctions, J. Am. Chem. Soc. 2014, 136, 16-19.

(28) Abu-Husein, T.; Schuster, S.; Egger, D. A.; Kind, M.; Santowski, T.; Wiesner, A.; Chiechi, R.; Zojer, E.; Terfort, A.; Zharnikov, M. The Effects of Embedded Dipoles in Aromatic Self-Assembled Monolayers, Adv. Funct. Mater. 2015, 25, 3943-3957.

(29) Hehn, I.; Schuster, S.; Wächter, T.; Abu-Husein, T.; Terfort, A.; Zharnikov, M.; Zojer, E. Employing X-ray Photoelectron Spectroscopy for Determining Layer Homogeneity in Mixed Polar Self-Assembled Monolayers, J. Phys. Chem. Lett. 2016, 7, 2994-3000. 
(30) Kovalchuk, A.; Abu-Husein, T.; Fracasso, D.; Egger, D. A.; Zojer, E.; Zharnikov, M.; Terfort, A.; Chiechi, R. C. Transition Voltages Respond to Synthetic Reorientation of Embedded Dipoles in Self-Assembled Monolayers. Chem. Sci. 2016, 7, 781-787.

(31) Kovalchuk, A.; Egger, D. A.; Abu-Husein, T.; Zojer, E.; Terfort, A.; Chiechi, R. C. Dipole-Induced Asymmetric Conduction in Tunneling Junctions Comprising Self-Assembled Monolayers. RSC Adv. 2016, 6, 69479-69483.

(32) Ahn, H.; Zharnikov, M.; Whitten, J. E. Abnormal Pinning of the Fermi and Vacuum Levels in Monomolecular Self-Assembled Films. Chem. Phys. Lett. 2006, 428, 283-287.

(33) Ge, Y.; Weidner, T.; Ahn, H.; Whitten, J. E.; Zharnikov, M. Energy Level Pinning in Self-Assembled Alkanethiol Monolayers. J. Phys. Chem. C 2009, 113, 4575-4583.

(34) Ballav, N.; Terfort, A.; Zharnikov, M. Mixing of Nonsubstituted and Partly Fluorinated Alkanethiols in a Binary Self-Assembled Monolayer. J. Phys. Chem. C 2009, 113, 3697-3706.

(35) Venkataraman, N. V.; Zuercher, S.; Rossi, A.; Lee, S.; Naujoks, N.; Spencer, N. D. Spatial Tuning of the Metal Work Function by Means of Alkanethiol and Fluorinated Alkanethiol Gradients. J. Phys. Chem. C 2009, 113, 5620-5628.

(36) Ratner, B. D.; Castner, D. G. Electron Spectroscopy for Chemical Analysis. In Surface Analysis—The Principle Techniques; Vickerman, J. C., Ed.; Wiley \& Sons: Chichester, 1997.

(37) Taucher, T. C.; Hehn, I.; Hofmann, O. T.; Zharnikov, M.; Zojer, E. Understanding Chemical versus Electrostatic Shifts in X-Ray Photoelectron Spectra of Organic Self-Assembled Monolayers. J. Phys. Chem. C 2016, 120, 3428-3437.

(38) Cabarcos, O. M.; Shaporenko, A.; Weidner, T.; Uppili, S.; Dake, L. S.; Zharnikov, M.; Allara, D. L. Physical and Electronic Structure Effects of Embedded Dipoles in Self-Assembled Monolayers: Characterization of Mid-Chain Ester Functionalized Alkanethiols on $\mathrm{Au}\{111\} . J$. Phys. Chem. C 2008, 112, 10842-10854.

(39) Natan, A.; Kronik, L.; Haick, H.; Tung, R. T. Electrostatic Properties of Ideal and Nonideal Polar Organic Monolayers: Implications for Electronic Devices. Adv. Mater. 2007, 19, 4103-4117.

(40) Heimel, G.; Rissner, F.; Zojer, E. Modeling the Electronic Properties of $\pi$-Conjugated Self-Assembled Monolayers. Adv. Mater. 2010, 22, 2494-2513.

(41) Parikh, A. N.; Allara, D. L. Quantitative Determination of Molecular Structure in Multilayered Thin Films of Biaxial and Lower Symmetry from 


\section{Photon Spectroscopies. I. Reflection Infrared Vibrational Spectroscopy J.}

Chem. Phys. 1992, 96, 927-945.

(42) Moulder, J. F.; Stickle, W. E.; Sobol, P. E.; Bomben, K. D. Handbook of

X-ray Photoelectron Spectroscopy, Chastian, J., Ed.; Perkin-Elmer Corp.: Eden Prairie, 1992.

(43) Kalinin, S.; Gruverman, A. Scanning Probe Microscopy: Electrical and

Electromechanical Phenomena at the Nanoscale. Part I. 1.4. Principles of Kelvin Probe Force

Microscopy; Springer: New York, 2007.

(44) Kresse, G.; Hafner, J. Ab Initio Molecular Dynamics for Liquid Metals. Phys. Rev. B 1993, 47, 558-561.

(45) Kresse, G.; Hafner, J. Ab Initio Molecular-Dynamics Simulation of the Liquid-MetalAmorphous-Semiconductor Transition in Germanium. Phys. Rev. B 1994, 49, 14251-14269.

(46) Kresse, G.; Furthmüller, J. Efficiency of Ab-Initio Total Energy Calculations for Metals and Semiconductors Using a Plane-Wave Basis Set. Comput. Mater. Sci. 1996, 6, 15-50.

(47) Kresse, G.; Furthmüller, J. Efficient Iterative Schemes for Ab Initio Total-Energy Calculations Using a Plane-Wave Basis Set. Phys. Rev. B 1996, 54, 11169-11186.

(48) Plimpton, S. Fast Parallel Algorithms for Short-Range Molecular Dynamics. J. Comput. Phys. 1995, 117, 1-19.

(49) Perdew, J. P.; Burke, K.; Ernzerhof, M. Generalized Gradient Approximation Made Simple. Phys. Rev. Lett. 1996, 77, 3865-3868.

(50) Ruiz, V. G.; Liu, W.; Zojer, E.; Scheffler, M.; Tkatchenko, A. Density-Functional Theory with Screened van Der Waals Interactions for the Modeling of Hybrid Inorganic-Organic Systems. Phys. Rev. Lett. 2012, 108, 146103.

(51) Al-Saidi, W. A.; Voora, V. K.; Jordan, K. D. An Assessment of the vdW-TS Method for Extended Systems. J. Chem. Theory Comput. 2012, 8, 1503-1513.

(52) Blöchl, P. E. Projector Augmented-Wave Method. Phys. Rev. B 1994, 50, 17953-17979.

(53) Kresse, G.; Joubert, D. From Ultrasoft Pseudopotentials to the Projector AugmentedWave Method. Phys. Rev. B 1999, 59, 1758-1775.

(54) Monkhorst, H. J.; Pack, J. D. Special Points for Brillouin-Zone Integrations. Phys. Rev. B 1976, 13, 5188-5192.

(55) Bučko, T.; Hafner, J.; Ángyán, J. G. Geometry Optimization of Periodic Systems Using Internal Coordinates. J. Chem. Phys. 2005, 122, 124508. 
(56) Fenter, P.; Eisenberger, P.; Liang, K. S. Chain-Length Dependence of the Structures and Phases of $\mathrm{CH}_{3}\left(\mathrm{CH}_{2}\right)_{\mathrm{n}-1} \mathrm{SH}$ Self-Assembled on $\mathrm{Au}(111)$. Phys. Rev. Lett. 1993, 70, 24472450 .

(57) Schreiber, F. Structure and Growth of Self-Assembling Monolayers. Prog. Surf. Sci. 2000, 65, 151-256.

(58) Chesneau, F.; Zhao, J.; Shen, C.; Buck, M.; Zharnikov, M. Adsorption of Long-Chain Alkanethiols on $\mathrm{Au}(111)$ - A Look from the Substrate by High Resolution X-ray Photoelectron Spectroscopy. J. Phys. Chem. C 2010, 114, 7112-7119.

(59) Neugebauer, J.; Scheffler, M. Adsorbate-Substrate and Adsorbate-Adsorbate Interactions of $\mathrm{Na}$ and $\mathrm{K}$ Adlayers on $\mathrm{Al}(111)$. Phys. Rev. B 1992, 46, 16067-16080.

(60) Köhler, L.; Kresse, G. Density Functional Study of CO on Rh(111). Phys. Rev. B 2004, $70,165405$.

(61) Lamont, C. L.; Wilkes, J. Attenuation Length of Electrons in Self-Assembled Monolayers of $n$-Alkanethiols on Gold. Langmuir 1999, 15, 2037-2042.

(62) Heister, K.; Johansson, L. S. O.; Grunze, M.; Zharnikov, M. A Detailed Analysis of the C 1s Photoemission of $n$-Alkanethiolate Films on Noble Metal Substrates, Surf. Sci. 2003, 529, 36-46.

(63) Zharnikov, M. High-Resolution X-Ray Photoelectron Spectroscopy in Studies of SelfAssembled Organic Monolayers. J. Electron Spectrosc. Relat. Phenom. 2010, 178, 380-393.

(64) Evans, S. D.; Ulman, A. Surface Potential Studies of Alkyl-Thiol Monolayers Adsborbed on Gold. Chem. Phys. Lett. 1990, 170, 462-466.

(65) Monti, O. L. A. Understanding Interfacial Electronic Structure and Charge Transfer: An Electrostatic Perspective. J. Phys. Chem. Lett. 2012, 3, 2342-2351.

(66) Otálvaro, D.; Veening, T.; Brocks G. Self-Assembled Monolayer Induced Au(111) and Ag(111) Reconstructions: Work Functions and Interface Dipole Formation, J. Phys. Chem. C 2012, 116, 7826-7837.

(67) Vieider, M. Molecular Dynamics Simulations of SAMs Formed by Ester Functionalized Alkylthiolates on Gold. MSc Thesis, The Graz University of Technology, March 2013. 
(68) Bagus, P. S.; Pacchioni, G.; Sousa, C.; Minerva, T.; Parmigiani, F. Chemical Shifts of the Core-Level Binding Energies for the Alkaline-Earth Oxides. Chem. Phys. Lett. 1992, 196, 641.

(69) Sousa, C.; Minerva, T.; Pacchioni, G.; Bagus, P.S.; Parmigiani F., Electrostatic and Chemical Bonding Contributions to the Cation Core Level Binding Energy Shifts in $\mathrm{MgO}, \mathrm{CaO}$, SrO, BaO. A Cluster Model Study, J. Electron Spectrosc. Relat. Phenom. 1993, 63, 189-205.

(70) Bagus, P. S.; Ilton, E. S.; Nelinc, C. J. The Interpretation of XPS Spectra: Insights into Materials Properties, Surf. Sci. Rep. 2013, 68, 273-304.

(71) Cornil, D.; Cornil, J. Work-Function Modification of the (111) Gold Surface upon Deposition of Self-Assembled Monolayers based on Alkanethiol Derivatives. J. Electron Spectrosc. Relat. Phenom. 2013, 189, 32-38.

(72) Thomas, J. C.; Goronzy, D. P.; Dragomiretskiy, K.; Zosso, D.; Gilles, J.; Osher, S. J.; Bertozzi, A. L.; Weiss, P. S. Mapping Buried Hydrogen-Bonding Networks. ACS Nano 2016, 10, 5446-5451. 
Table 1: SAM Precursor Molecules of This Study and Their Abbreviations

\begin{tabular}{|c|c|}
\hline Compound & Abbreviation \\
\hline $\mathrm{SH}\left(\mathrm{CH}_{2}\right)_{11} \mathrm{CH}_{3}$ & $\mathrm{C} 12$ \\
\hline $\mathrm{SH}\left(\mathrm{CH}_{2}\right){ }_{15} \mathrm{CH}_{3}$ & $\mathrm{C} 16$ \\
\hline $\mathrm{SH}\left(\mathrm{CH}_{2}\right)_{17} \mathrm{CH}_{3}$ & $\mathrm{C} 18$ \\
\hline $\mathrm{SH}\left(\mathrm{CH}_{2}\right){ }_{19} \mathrm{CH}_{3}$ & $\mathrm{C} 20$ \\
\hline $\mathrm{SH}\left(\mathrm{CH}_{2}\right)_{21} \mathrm{CH}_{3}$ & $\mathrm{C} 22$ \\
\hline $\mathrm{SH}\left(\mathrm{CH}_{2}\right)_{5} \mathrm{COO}\left(\mathrm{CH}_{2}\right)_{9} \mathrm{CH}_{3}$ & C5EC10 \\
\hline $\mathrm{SH}\left(\mathrm{CH}_{2}\right)_{10} \mathrm{COO}\left(\mathrm{CH}_{2}\right)_{4} \mathrm{CH}_{3}$ & C10EC5 \\
\hline $\mathrm{SH}\left(\mathrm{CH}_{2}\right){ }_{10} \mathrm{COO}\left(\mathrm{CH}_{2}\right)_{9} \mathrm{CH}_{3}$ & C10EC10 \\
\hline $\mathrm{SH}\left(\mathrm{CH}_{2}\right){ }_{10} \mathrm{COO}\left(\mathrm{CH}_{2}\right){ }_{14} \mathrm{CH}_{3}$ & C10EC15 \\
\hline $\mathrm{SH}\left(\mathrm{CH}_{2}\right)_{15} \mathrm{COO}\left(\mathrm{CH}_{2}\right)_{4} \mathrm{CH}_{3}$ & C15EC5 \\
\hline $\mathrm{SH}\left(\mathrm{CH}_{2}\right){ }_{15} \mathrm{COO}\left(\mathrm{CH}_{2}\right){ }_{9} \mathrm{CH}_{3}$ & C15EC10 \\
\hline $\mathrm{SH}\left(\mathrm{CH}_{2}\right)_{20} \mathrm{COO}\left(\mathrm{CH}_{2}\right)_{4} \mathrm{CH}_{3}$ & C20EC5 \\
\hline $\mathrm{SH}\left(\mathrm{CH}_{2}\right){ }_{10} \mathrm{OOC}\left(\mathrm{CH}_{2}\right)_{9} \mathrm{CH}_{3}$ & $\mathrm{C} 10 \mathrm{E}^{*} \mathrm{C} 10$ (reverse ester) \\
\hline $\mathrm{SH}\left(\mathrm{CH}_{2}\right){ }_{10} \mathrm{COO}\left(\mathrm{CH}_{2}\right)_{4} \mathrm{COO}\left(\mathrm{CH}_{2}\right)_{4} \mathrm{CH}_{3}$ & C10EC4EC5 (double ester) \\
\hline
\end{tabular}




\section{TOC Graphic}

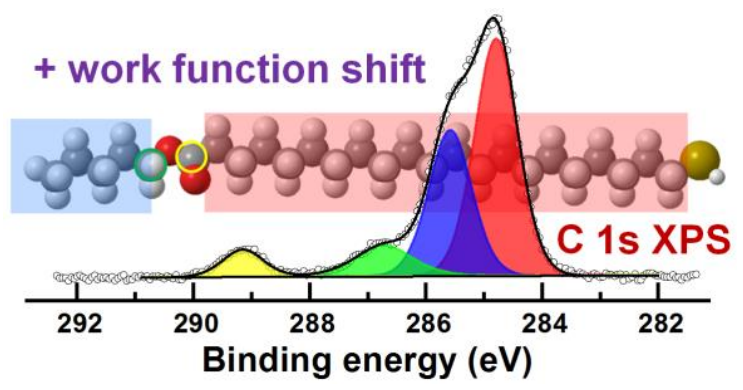

